\title{
Functional Plasticity of Gamma Delta T Cells and Breast Tumor Targets in Hypoxia
}

OPEN ACCESS

Edited by:

Kenth Gustafsson,

University College London,

United Kingdom

Reviewed by:

Tomasz Zal,

University of Texas MD Anderson Cancer Center,

United States

Christoph Wülfing,

University of Bristol,

United Kingdom

*Correspondence:

Gabrielle M. Siegers

siegers@ualberta.ca

Specialty section: This article was submitted to T Cell Biology,

a section of the journal

Frontiers in Immunology

Received: 07 March 2018

Accepted: 01 June 2018

Published: 15 June 2018

Citation:

Siegers GM, Dutta I, Lai R and Postovit L-M (2018) Functional

Plasticity of Gamma

Delta T Cells and Breast Tumor

Targets in Hypoxia.

Front. Immunol. 9:1367.

doi: 10.3389/fimmu.2018.01367

\author{
Gabrielle M. Siegers ${ }^{1,2 *}$, Indrani Dutta ${ }^{1}$, Raymond Lai ${ }^{1}$ and Lynne-Marie Postovit ${ }^{1,2}$ \\ ${ }^{1}$ Department of Oncology, University of Alberta, Edmonton, AB, Canada, ${ }^{2}$ Department of Anatomy and Cell Biology, \\ Robarts Research Institute, Western University, London, ON, Canada
}

Interactions between immune and tumor cells in the tumor microenvironment (TME) often impact patient outcome, yet remain poorly understood. In addition, the effects of biophysical features such as hypoxia [low oxygen $\left(\mathrm{O}_{2}\right)$ ] on cells within the TME may lead to tumor evasion. Gamma delta T cells ( $\gamma \delta$ Tcs) naturally kill transformed cells and are therefore under development as immunotherapy for various cancers. Clinical trials have proven the safety of $\gamma \delta \mathrm{Tc}$ immunotherapy and increased circulating $\gamma \delta \mathrm{Tc}$ levels correlate with improved patient outcome. Yet, the function of $\gamma \delta$ Tc tumor infiltrating lymphocytes in human breast cancer remains controversial. Breast tumors can be highly hypoxic, thus therapy must be effective under low $\mathrm{O}_{2}$ conditions. We have found increased infiltration of $\gamma \delta \mathrm{Tc}$ in areas of hypoxia in a small cohort of breast tumors; considering their inherent plasticity, it is important to understand how hypoxia influences $\gamma \delta$ Tc function. In vitro, the cell density of expanded primary healthy donor blood-derived human $\gamma \delta T_{c}$ decreased in response to hypoxia $\left(2 \% \mathrm{O}_{2}\right)$ compared to normoxia $\left(20 \% \mathrm{O}_{2}\right)$. However, the secretion of macrophage inflammatory protein $1 \alpha(\mathrm{MIP} 1 \alpha) / \mathrm{MIP} 1 \beta$, regulated on activation, normal $T$ cell expressed and secreted (RANTES), and CD40L by $\gamma \delta$ Tc were increased after $40 \mathrm{~h}$ in hypoxia compared to normoxia concomitant with the stabilization of hypoxia inducible factor 1-alpha protein. Mechanistically, we determined that natural killer group 2, member D (NKG2D) on $\gamma \delta T c$ and the NKG2D ligand MHC class I polypeptide-related sequence A (MICA)/B on MCF-7 and T47D breast cancer cell lines are important for $\gamma \delta$ TC cytotoxicity, but that MIP1 $\alpha$, RANTES, and CD40L do not play a direct role in cytotoxicity. Hypoxia appeared to enhance the cytotoxicity of $\gamma \delta \mathrm{Tc}$ such that exposure for $48 \mathrm{~h}$ increased cytotoxicity of $\gamma \delta \mathrm{Tc}$ against breast cancer cells that were maintained in normoxia; conversely, breast cancer lines incubated in hypoxia for $48 \mathrm{~h}$ prior to the assay were largely resistant to $\gamma \delta$ Tc cytotoxicity. MICA/B surface expression on both MCF-7 and T47D remained unchanged upon exposure to hypoxia; however, ELISAs revealed increased MICA shedding by MCF-7 under hypoxia, potentially explaining resistance to $\gamma \delta$ Tc cytotoxicity. Despite enhanced $\gamma \delta$ Tc cytotoxicity upon pre-incubation in hypoxia, these cells were unable to overcome hypoxia-induced resistance of MCF-7. Thus, such resistance mechanisms employed by breast cancer targets must be overcome to develop more effective $\gamma \delta$ Tc immunotherapies.

Keywords: gamma delta T cells, plasticity, hypoxia, breast cancer, tumor evasion, MHC class I polypeptide-related sequence $A$ 


\section{INTRODUCTION}

Low oxygen $\left(\mathrm{O}_{2}\right)$ levels (hypoxia) characterize the microenvironment of many solid tumors, occurring as a consequence of structurally disorganized blood vessels and tumor growth that exceeds the rate of vascularization. Hypoxia is common within breast cancers, which have a median $\mathrm{O}_{2}$ concentration of $1.4 \%$, as compared to $\sim 9.3 \%$ for normal breast tissue (1). In response to hypoxia, cells express genes that are essential for their survival. In tumor cells, this $\mathrm{O}_{2}$-regulated gene expression leads to more aggressive phenotypes, including those that increase the ability of cells to resist therapy, recruit a vasculature and metastasize (2-4). Accordingly, there is a growing body of evidence correlating tumor hypoxia with poor clinical outcome for patients with a variety of cancers $(5-7), \mathrm{O}_{2}$ availability has also been shown to regulate immune editing, allowing cancer cells to evade the immune system via a variety of mechanisms (8). For example, hypoxia upregulates hypoxia inducible factor 1-alpha (HIF1 $\alpha$ )-dependent ADAM10 expression resulting in MHC class I polypeptide-related sequence A (MICA) shedding from the surface and decreased lysis of tumor cells (9). While many studies have focused on myeloid-derived suppressor cells or conventional CD8+ T cells (8), so far none have considered the impact of tumor hypoxia on gamma delta $\mathrm{T}$ cells $(\gamma \delta \mathrm{Tcs})$.

While $\gamma \delta \mathrm{Tc}$ kill cancer cell lines, derived from both hematological and solid tumors alike [reviewed in Ref. (10)], it is unclear whether they are still active cancer killers when confronted with the harsh and immunosuppressive tumor microenvironment (TME) (10-13). We have focused on breast cancer, since there have been conflicting reports in the literature with respect to $\gamma \delta \mathrm{Tc}$ function in this disease. While in vitro studies clearly show that $\gamma \delta \mathrm{Tc}$ are able to kill breast cancer cell lines MDA-MB231, MCF-7, and T47D (14-16), it is unclear as to whether $\gamma \delta \mathrm{Tc}$ retain their cytotoxic properties once exposed to the breast tumor TME (11).

Here, we set out to determine how $\gamma \delta \mathrm{Tc}$ behave under low $\mathrm{O}_{2}$, a TME factor likely encountered by $\gamma \delta \mathrm{Tc}$ in many malignancies. Carbonic anhydrase IX (CAIX) is a transmembrane protein that catalyzes the reversible hydration of carbon dioxide. It is expressed in response to hypoxia and is thus used as a surrogate marker for hypoxia (17). High CAIX expression indicates poor prognosis in many cancers, including breast cancer (18-20). Breast cancer cell lines express MICA, a ligand for the natural killer group 2, member D (NKG2D) receptor expressed by $\gamma \delta \mathrm{Tc}$ and implicated in $\gamma \delta \mathrm{Tc}$ cytotoxicity (21-25). Thus, we have further explored

Abbreviations: BP, band pass; CAIX, carbonic anhydrase IX; CalAM, Calcein AM; CD40L, CD40 ligand (or CD154); E:T, effector:target ratio; ER, estrogen receptor; FBS, fetal bovine serum; FMO, fluorescence minus one; $\gamma \delta \mathrm{Tcs}$, gamma delta T cells; HIF1 $\alpha$, hypoxia inducible factor 1-alpha; HRP, horseradish peroxidase; IL, interleukin; LP, long pass; MICA, MHC class I polypeptide-related sequence A; MIP1 $\alpha$, macrophage inflammatory protein $1 \alpha$ [or CCL3 $=$ chemokine $(\mathrm{C}-\mathrm{C}$ motif $)$ ligand 3]; MFI, median fluorescence intensity; NKG2D, natural killer group 2, member D; $\mathrm{O}_{2}$, oxygen; PBMCs, peripheral blood mononuclear cells; PBS, phosphate buffered saline; PIC, protease and phosphatase inhibitor cocktail; PR, progesterone receptor; RANTES, regulated on activation, normal T cell expressed and secreted (or CCL5); TBST, tris-buffered saline plus $0.05 \%$ Tween-20; TCR, T cell antigen receptor; TIL, tumor infiltrating lymphocytes; TME, tumor microenvironment; TNF $\alpha$, tumor necrosis factor alpha; ZA, Zombie Aqua fixable viability dye. the integral role for NKG2D/MICA in $\gamma \delta$ Tc cytotoxicity against breast cancer cell lines under hypoxia and normoxia.

Since $\gamma \delta \mathrm{Tc}$ are being developed for cancer immunotherapy (26-31), and have shown both safety and even some efficacydespite advanced disease stage - in a Phase I trial for breast cancer (32), it is imperative that we learn how the TME impacts the function of $\gamma \delta \mathrm{Tc}$ infiltrating breast and other solid tumors.

\section{MATERIALS AND METHODS}

\section{Ethics Statement}

This study was carried out in accordance with the recommendations of the Research Ethics Guidelines, Health Research Ethics Board of Alberta-Cancer Committee with written informed consent from all subjects. All subjects gave written informed consent in accordance with the Declaration of Helsinki. The protocol was approved by the Health Research Ethics Board of Alberta-Cancer Committee.

\section{Patients and Tissues}

We assessed 17 surgically resected breast tumors from cancer patients diagnosed at the Cross Cancer Institute, Edmonton, AB, Canada from 1997 to 1998. Patient and tumor characteristics are listed in Table 1.

\section{Immunohistochemistry}

Anti-human T cell antigen receptor (TCR) $\delta$ staining was performed as reported (33). Briefly, $4.5 \mu \mathrm{m}$ serial sections from formalin-fixed paraffin-embedded tumors were melted on a slide warmer at $60^{\circ} \mathrm{C}$ for a minimum of $10 \mathrm{~min}$ followed by de-paraffinization using a

TABLE 1 | Characteristics of breast cancer cohort.

\begin{tabular}{|c|c|c|}
\hline$n=17$ & $n(\%)$ & Median (range) \\
\hline Age at diagnosis & & $51(40-69)$ \\
\hline \multicolumn{3}{|l|}{ Histology } \\
\hline Invasive ductal carcinoma & $14(82)$ & \\
\hline Invasive non-ductal tubular & $1(6)$ & \\
\hline Invasive non-ductal mucinous & $1(6)$ & \\
\hline Non-invasive & $1(6)$ & \\
\hline \multicolumn{3}{|l|}{ Tumor size (cm) } \\
\hline$<2$ & $11(65)$ & $1.4(0.2-5.5)$ \\
\hline $2-5$ & $4(24)$ & \\
\hline$>5$ & $1(6)$ & \\
\hline Not specified & $1(6)$ & \\
\hline \multicolumn{3}{|l|}{ Tumor grade } \\
\hline $1 / 3$ & $4(24)$ & \\
\hline $2 / 3$ & $5(29)$ & \\
\hline $3 / 3$ & $8(47)$ & \\
\hline \multicolumn{3}{|l|}{ Nodal status } \\
\hline Positive & $9(53)$ & \\
\hline Negative & $8(47)$ & \\
\hline \multicolumn{3}{|l|}{ Estrogen receptor } \\
\hline Positive & $12(71)$ & \\
\hline Negative & $3(18)$ & \\
\hline Not available & $2(12)$ & \\
\hline \multicolumn{3}{|l|}{ Progesterone receptor } \\
\hline Positive & $10(59)$ & \\
\hline Negative & $5(29)$ & \\
\hline Not available & $2(12)$ & \\
\hline
\end{tabular}


fresh Xylenes (Thermo Fisher Scientific, Burlington, ON, Canada) bath. Sections were then hydrated with a series of graded ethanol $(100,95,70$, and $60 \%)$ followed by brief incubation in water, then tris-buffered saline plus $0.05 \%$ Tween-20 (TBST). Antigen retrieval was performed at $100^{\circ} \mathrm{C}$ for $20 \mathrm{~min}$ in target retrieval solution $\mathrm{pH}$ 9 (DAKO North America, Carpinteria, CA, USA). After cooling to room temperature, tissues were circled with an ImmEdge pen (Vector Laboratories, Burlingame, CA, USA) and blocked with Peroxidase Block (DAKO) for $5 \mathrm{~min}$. Slides were washed in TBST for 5 min then blocked with Protein Block Serum Free (DAKO) for 10 min. Protein block was gently removed and replaced with 1:150 dilution of mouse monoclonal anti-human TCR $\delta$ antibody (clone H-41, Santa Cruz Biotechnology, Dallas, TX, USA) or 1:50 dilution of rabbit monoclonal anti-human CAIX [clone EPR4151(2), abcam, Cambridge, MA, USA] or corresponding isotype control diluted to the same antibody concentration; all dilutions were made in antibody diluent (DAKO). Known positive controls and isotype controls were included with each batch to ensure quality control of staining. Sections were incubated in a humidified chamber for $30 \mathrm{~min}$ at $25^{\circ} \mathrm{C}$. Slides were then rinsed and washed five times in TBST for $5 \mathrm{~min}$. Slides were then incubated with $100 \mu \mathrm{l}$ secondary antibody, labeled polymer-horseradish peroxidase (HRP) anti-mouse or-HRP anti-rabbit (DAKO), for $60 \mathrm{~min}$ at room temperature in the humidified chamber. Washing was done as before, and then slides were treated with 75-100 $\mu 1$ 3,3' -diaminobenzidine chromogen solution (DAKO) for 8-10 min before the reaction was stopped by rinsing in water. Hematoxylin (DAKO) counterstaining was performed, slides were rinsed in water and then dehydrated using a series of graded ethanol $(60,70,95$, and $100 \%)$. Slides were then cleared with Xylenes, dried and coverslips mounted with VectaMount permanent mounting medium (Vector Laboratories).

\section{Assessment of CAIX Expression and $\gamma \delta \mathrm{TC}$ Infiltration}

Light microscopy and semi-quantitative scoring for CAIX was performed by a single pathologist; scores were 0 , no staining; 1 , weak and/or very focal staining; $2+$, strong but focal staining; and 3, strong and extensive staining. Serial sections stained for $\mathrm{TCR} \gamma \delta$ and CAIX were scanned. Areas of CAIX-positivity and negativity were defined, and images from slides superimposed to enable counting of $\gamma \delta \mathrm{Tc}$ in CAIX-positive and -negative areas. Five consecutive areas within each region were quantified for the frequency of $\gamma \delta \mathrm{Tc}$ infiltration.

\section{Primary $\gamma \delta \mathrm{TC}$}

We established and maintained primary human $\gamma \delta$ Tc cultures as described (34). Briefly, healthy donor blood was diluted with phosphate buffered saline (PBS) and peripheral blood mononuclear cells (PBMCs) isolated using density gradient separation (Lymphoprep, Stem Cell Technologies, Vancouver, BC, Canada). PBMCs were cultured in a humidified atmosphere at $37^{\circ} \mathrm{C}$ with $5 \% \mathrm{CO}_{2}$ at $1 \times 10^{6}$ cells $/ \mathrm{ml}$ in RPMI complete medium containing $1 \mu \mathrm{g} / \mathrm{ml}$ Concanavalin A (Sigma-Aldrich, Oakville, ON, Canada), $10 \%$ fetal bovine serum (FBS), 1× MEM NEAA, 10 mM HEPES, 1 mM Sodium Pyruvate (all Invitrogen, Burlington, ON, Canada), and $10 \mathrm{ng} / \mathrm{ml}$ recombinant human interleukin (IL)-2 and IL-4 (Miltenyi Biotec, Auburn, CA, USA). Cells were counted and viability assessed via Trypan Blue Exclusion Assay (Invitrogen/ Thermo Fisher Scientific, Waltham, MA, USA); fresh medium and cytokines added to adjust density to $1 \times 10^{6}$ cells $/ \mathrm{ml}$ every 3-4 days. After 1 week, $\alpha \beta$ T cells were labeled with anti-TCR $\alpha \beta$ $\mathrm{PE}$ antibodies (BioLegend, San Diego, CA, USA) and anti-PE microbeads (Miltenyi Biotec), and depleted after filtering ( $50 \mu \mathrm{m}$ Cell Trics filter, Partec, Görlitz, Germany) and passing over an LD depletion column (Miltenyi Biotec). $\gamma \delta$ Tcs, which did not bind to the column, were further cultured in complete medium plus cytokines (as above). For cytotoxicity and blocking experiments, $\gamma \delta$ Tc cultures were used on days 19-21, as they were most cytotoxic then. Some hypoxia experiments were done at earlier time points. Donor cultures are identified as follows: donor number culture letter-culture day; thus, $7 \mathrm{~B}-13=$ the second culture derived from donor 7 on day 13. Culture purities and subset compositions are shown in Table S1 in Supplementary Material.

\section{Breast Cancer Cell Lines}

Human breast carcinoma cell lines, MCF-7 and T47D, were obtained from the American Type Culture Collection (ATCC, Manassas, VA, USA) and maintained as per ATCC guidelines. For surface marker staining of breast cancer cell lines, cells were harvested by washing with PBS followed by dissociation in Accutase (Sigma-Aldrich) for $20 \mathrm{~min}$ at $37^{\circ} \mathrm{C}$.

\section{Hypoxia Experiments}

To examine the effects of hypoxia, cells were cultured in $\mathrm{O}_{2}$ concentrations as indicated for $40-48 \mathrm{~h}$ using an X3 Xvivo Closed Incubation System (BioSpherix). After incubation under normoxic or hypoxic conditions, cell culture supernatants were collected, chilled on ice, and then frozen at $-80^{\circ} \mathrm{C}$ until further analysis; harvested cells were used in cytotoxicity assays or stained for flow cytometric analysis. In some cases, cells were cold harvested, pellets frozen on dry ice, and stored at $-80^{\circ} \mathrm{C}$ until lysis for Western blotting.

\section{Flow Cytometry Antibodies}

For surface marker staining of $\gamma \delta \mathrm{Tc}$, the following anti-human antibodies from BioLegend, unless otherwise indicated, were

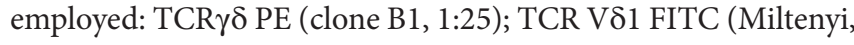
clone REA173, 1:10); TCR V82 PerCP (clone B6, 1:25); NKG2D APC (BD Biosciences, Mississauga, ON, Canada, 1:25); CD56 FITC (clone MEM-188, 1:5); CD69 AF700 (clone FN50, 1:4); CD94 FITC (clone DX22, 1:5); CD95 APC (clone DX2, 1:100); HLA ABC PE (clone W6/32, 1:10); FasL PE (clone NOK-1, 1:5); and CD40L APC (clone 24-31, 1:5).

Anti-human MICA/B PE (BioLegend, clone 6D4, $0.1 \mu \mathrm{g}$ ) was used to stain breast cancer cell lines.

\section{Surface Marker Staining}

Gamma delta $\mathrm{T}$ cell and breast cancer cell lines were adjusted to $10 \times 10^{6}$ cells $/ 1 \mathrm{ml}$, stained with $1 \mu \mathrm{l} / 10^{6}$ cells Zombie Aqua fixable viability dye in PBS (ZA, BioLegend) for 15-30 min at 
room temperature in the dark. $\gamma \delta T c$ were stained directly with fluorochrome-conjugated antibodies diluted in FACS buffer [PBS containing 1\% FBS and $2 \mathrm{mM}$ EDTA (Invitrogen)] as indicated above. Breast cancer cell lines at $10 \times 10^{6} \mathrm{cells} / \mathrm{ml}$ were blocked in FACS buffer containing $50 \mu \mathrm{l} / \mathrm{ml}$ Trustain FcX (BioLegend) and incubated on ice for $30 \mathrm{~min}$ prior to antibody incubation. After blocking, cells were centrifuged and supernatants removed, leaving $10 \mu \mathrm{l} \mathrm{FACS} \mathrm{buffer} \mathrm{plus} \mathrm{block} / 10^{6}$ cells. Antibodies and FACS buffer were added to $20 \mu \mathrm{l}$, and cells incubated on ice 15-20 min followed by washing. All cells were fixed in FACS buffer containing $2 \%$ paraformaldehyde (Sigma-Aldrich), stored at $4^{\circ} \mathrm{C}$ and acquired within 1 week.

\section{Flow Cytometer Specifications}

Cells were analyzed using a FACS CANTO II (Becton Dickinson, Mississauga, ON, Canada) equipped with an air-cooled 405-nm solid state diode, $30 \mathrm{~mW}$ fiber power output violet laser, with $450 / 50$ and 510/50 band pass (BP) [502 long pass (LP) detector]; a 488-nm solid state, 20-mW blue laser with 530/30 BP (502 LP), 585/42 BP (556 LP), 670 LP (655 LP), and 780/60 BP (735 LP) filters; and a 633-nm HeNe, 17-mW red laser with 660/20 BP and 780/60 BO (735 LP) filters. Calibration was performed with CS\&T beads (Becton Dickenson, Mississauga, ON, Canada). Live singlets were gated based on forward and side-scatter properties. Fluorescence minus one (FMO) controls were used to set gates. Analysis was performed using ${\text { Flow } \mathrm{Jo}^{\odot}}^{\odot}$ software (Tree Star, Ashland, OR, USA, Version 10.0.8r1).

\section{Cytokine Arrays}

The Proteome Profiler Human Cytokine Array Kit, Panel A (R\&D Systems, Minneapolis, MN, USA) was used to detect proteins secreted by $\gamma \delta T c$ cultured under normoxic or hypoxic conditions. Undiluted culture supernatants were used in these assays, which were carried out according to the manufacturer's instructions. Analysis of resulting films was done as follows: pixel intensities were measured using FIJI software (ImageJ Version 2.0.0-rc-15/ $1.49 \mathrm{~m}$ ) using a consistent circular region of interest; measured values from duplicate spots were subtracted from 255 . The average intensity from the two negative spots was subtracted from all values to obtain net values. The intensities of the six reference spots (positive controls) were averaged and a multiplier was defined for each array (normalized to the array with the lowest pixel intensity). Values were adjusted accordingly and then values for the duplicates were averaged. Finally, ratios were calculated for each cytokine, normalized to normoxia.

\section{ELISAs}

1-2 $\mathrm{ml}$ aliquots of culture supernatants stored at $-80^{\circ} \mathrm{C}$ were thawed on ice. Halt ${ }^{\mathrm{TM}}$ Protease and Phosphatase Inhibitor Cocktail (PIC, Thermo Fisher Scientific) was added to samples prior to use in ELISAs or further storage at $4^{\circ} \mathrm{C}$. The following ELISA kits were used: ELISA MAX Deluxe regulated on activation, normal T cell expressed and secreted (RANTES/CCL5) (BioLegend), Human macrophage inflammatory protein $1 \alpha(\mathrm{MIP} 1 \alpha)$ and Human CD40L Quantikine ELISA kits (R\&D Systems), and Human MICA ELISA Kit (abcam). For RANTES and CD40L ELISAs, culture supernatant samples were diluted up to 16 -fold to obtain readings within range $(1: 2,1: 4,1: 8,1: 16)$. For MIP1 $\alpha$ ELISAs, samples were diluted up to 1:20. For MICA ELISAs, culture supernatants stored at $-80^{\circ} \mathrm{C}$ were thawed overnight in at $4^{\circ} \mathrm{C}$, then $4 \mathrm{ml}$ applied to Amicon Ultra- 4 $10 \mathrm{~K}$ spin columns (Merck-Millipore, Carrigtwohill, Ireland) that were subsequently centrifuged at $3,000 \mathrm{~g}$ for $2 \mathrm{~h}$ at $12^{\circ} \mathrm{C}$. Concentrated media was then transferred into $1.5 \mathrm{ml}$ Eppendorf tubes and diluted to 200 and $20 \mu \mathrm{l}$ of a 1:10 dilution of PIC were added. For the ELISA, $100 \mu \mathrm{l}$ per well were assayed in duplicate. All ELISAs were done according to the manufacturer's instructions. Absorbance at 450 and $550 \mathrm{~nm}$ was measured using a FLUOstar Omega plate reader (BMG Labtech, Offenburg, Germany) with Omega Software version 5.11. The difference linear regression fit of the standard curve was used for concentration calculations. ELISA data were normalized to $\gamma \delta$ Tc cell numbers and culture volumes.

\section{Immunoblotting}

Cell lysates were prepared by mixing $\gamma \delta \mathrm{Tc}$ with M-PER Mammalian Protein Extraction Reagent (Thermo Fisher Scientific) containing PIC at $10 \mu \mathrm{l}$ lysis buffer per million $\gamma \delta$ Tc followed by incubation at room temperature for $10 \mathrm{~min}$. Lysates were then centrifuged at $13,000 \mathrm{rpm}$ for $15 \mathrm{~min} 4^{\circ} \mathrm{C}$, after which supernatants were transferred to fresh tubes and $5 \times$ reducing sample buffer [0.0625 M Tris/HCl pH6.8, 2\% SDS, 20\% glycerol, $0.05 \%$ $\beta$-mercaptoethanol, $0.025 \%$ (w/v) Bromophenol Blue] added. Samples were boiled $5 \mathrm{~min}$, cooled, and briefly centrifuged in a benchtop centrifuge prior to running on 10 or $12 \%$ SDS-PAGE gels. Proteins were transferred onto Immobilon-FL PVDF membranes (Millipore) using the Trans-Blot Turbo Transfer System (Bio-Rad, Mississauga, ON, Canada). The high molecular weight (MW) program was used when transferring proteins for HIF $1 \alpha$ detection. Otherwise, the mixed MW program was used. Membranes were blocked $40 \mathrm{~min}$ in $3 \%$ milk in TBST, followed by overnight incubation in primary antibody baths at $4^{\circ} \mathrm{C}$. After washing, membranes were incubated with the corresponding species-specific HRP-labeled secondary antibody for $1 \mathrm{~h}$, followed by further washing and then detection using Clarity $^{\mathrm{TM}}$ Western ECL Substrate (Bio-Rad). Primary antibodies were diluted in PBS containing 2\% bovine serum albumin and $0.05 \%$ sodium azide at the following dilutions: 1:500 mouse anti-human HIF-1 $\alpha$ (clone MOP1, BD Biosciences); 1:2,000 goat anti-human CCL3/MIP1 $\alpha$ (R\&D Systems); 1:1,000 mouse antihuman/primate CCL5/RANTES (Clone \#21418, R\&D Systems); 1:500 mouse anti-human CD40 ligand/TNFSF5 (Clone \#40804, R\&D Systems); 1:2,000 rabbit anti-human $\beta$-Actin (Cell Signaling Technologies, Danvers, MA, USA). Secondary antibodies were diluted in blocking buffer as follows: 1:10,000 goat anti-mouse IgG HRP (Bio-Rad); 1:20,000 goat anti-rabbit IgG HRP (BioRad); and 1:1,000 donkey anti-goat IgG HRP (R\&D Systems).

\section{Quantification of Bands on Western Blots}

Band intensities for CD40L, MIP1 $\alpha$, and RANTES were measured using FIJI software (ImageJ Version 2.0.0-rc-15/1.49m) on converted grayscale images using consistent rectangular regions of interest. Measured values for bands and background (region of same size beneath each band) were subtracted from 255, then background was subtracted from bands to obtain net values for 
protein bands of interest and loading control bands (actin). The ratios of protein bands to loading control bands were then calculated. In the case of CD40L and RANTES, these values were multiplied by 10 to obtain values between 0.1 and 10 . For calculation of induction, hypoxia values were divided by normoxia values, and average values for each protein were plotted. Calculations were done in Microsoft Excel version 15.3 (Microsoft, Redmond, WA, USA).

\section{Cytotoxicity Assays}

Target Cell Labeling With Calcein AM (CalAM)

As per the manufacturer's instructions, target cells were labeled with $5 \mu \mathrm{M}$ CalAM (Invitrogen/Thermo Fisher Scientific). Cells were diluted to 30,000 cells $/ 100 \mu \mathrm{l}$ medium for cytotoxicity assays.

\section{Blocking Antibodies}

The following anti-human antibodies were used: LEAF purified anti-NKG2D (BioLegend, Clone 1D11); anti-human CCL3/MIP1 $\alpha$ (R\&D Systems); anti-human/primate CCL5/RANTES (Clone \#21418, R\&D Systems); and anti-human CD40 ligand/TNFSF5 (Clone \#40804, R\&D Systems). Mouse IgG (Sigma-Aldrich) was used as a control.

\section{Blocking/Cytotoxicity Assay}

For blocking and cytotoxicity assays, $6 \times 10^{6}$ cells $/ \mathrm{ml} \gamma \delta \mathrm{Tc}$ cells were re-suspended in complete medium: RPMI 1640 plus $10 \%$ heat-inactivated FBS; 10 mM HEPES; $1 \times$ MEM NEAA; $1 \mathrm{mM}$ sodium pyruvate; $50 \mathrm{U} / \mathrm{ml}$ penicillin-streptomycin; and $2 \mathrm{mM}$ L-glutamine, all purchased from Invitrogen. Blocking antibodies were added at $6 \mu \mathrm{g} \mathrm{mAb}$ per $600 \mu \mathrm{l}$ cell suspension/test in Eppendorf tubes, then plated at $100 \mu \mathrm{l} /$ well in a 96-well roundbottomed plate and incubated at $37^{\circ} \mathrm{C}$ for $30 \mathrm{~min}$. Thereafter, $100 \mu \mathrm{l}$ CalAM-labeled targets were added. For cytotoxicity assays, the effector:target $(\mathrm{E}: \mathrm{T})$ ratio is indicated; blocking assays were done at 20:1. Co-cultures were incubated at $37^{\circ} \mathrm{C}$ for $4 \mathrm{~h}$, after which plates were centrifuged and supernatants transferred to black clear-bottom 96-well (flat) plates (Costar, VWR International, Edmonton, AB, Canada). CalAM fluorescence was then detected on a FLUOstar Omega, BMG labtech fluorimeter. Controls were untreated and IgG-treated cells (for blocking assays), CalAM-labeled target cells incubated alone (spontaneous release) as well as $0.05 \%$ Triton-X 100 (Thermo Fisher Scientific)-treated cells (maximum release). The calculation for percent lysis is: [(test-spontaneous release)/(maximumspontaneous release) $] \times 100 \%$.

\section{Statistics}

The following tests were used to determine significance: paired one-tailed Student's $t$-tests [Figures 2A,B only, Microsoft Excel version 15.3 (Microsoft, Redmond, WA, USA)]; paired twotailed Student's $t$-tests [Figures 2C-K, Prism 7.0 for Mac OSX (GraphPad Software, San Diego, CA, USA)]; one-way ANOVA analysis (Figure 4, Prism); and Shapiro-Wilk normality tests followed by two-way ANOVA (Figure 1E, 3, 5, 6, Prism). Sidak's pairwise multiple comparison post hoc tests were performed alongside ANOVA analyses. The threshold for significance was set at $P<0.05$; asterisks indicate degrees of significance as defined in the figure legends.

\section{RESULTS}

\section{$\gamma \delta \mathrm{Tc}$ Can Be Found in Hypoxic Regions in Breast Cancer Cases}

In order to determine whether $\gamma \delta \mathrm{Tc}$ are present in areas of hypoxia in breast tumors, we performed immunohistochemistry to detect the hypoxia marker CAIX and $\gamma \delta \mathrm{Tc}$ using single stains of serial sections from a panel of 17 breast tumors (Table 1). Examples from one case (case 14) are shown (Figures 1A-D), including images of a CAIX-positive region (Figure 1A), an area with no appreciable CAIX positivity (Figure 1B), and increased magnification of $\gamma \delta \mathrm{Tc}$ found in the same region depicted in Figure 1A (Figure 1C) and Figure 1B (Figure 1D). Of these 17 cases, $47 \%$ (8/17) stained positively for CAIX. In CAIX-negative cases, there was little $\gamma \delta \mathrm{Tc}$ infiltration; however, when $\gamma \delta \mathrm{Tc}$ were quantified in CAIX-positive versus CAIX-negative areas of breast tumors, $\gamma \delta \mathrm{Tc}$ frequency was greater in hypoxic regions, significantly so in three cases in particular (Figure 1E, cases 13, 14, and 17, $P<0.0001$ ). Images for cases 13 and 17 are in Figure S1 in Supplementary Material. In our cohort, $71 \%$ (12/17) of tumors were estrogen receptor positive $(\mathrm{ER}+)$; most $\mathrm{ER}+$ cases were CAIX-negative (Figure 1E, ER status indicated below case numbers).

\section{Exposure to Hypoxia Reduces $\gamma \delta \mathrm{Tc}$ Density}

Given the co-localization of $\gamma \delta \mathrm{Tc}$ and CAIX in breast tumors, we measured the effects of hypoxia on $\gamma \delta \mathrm{Tc}$ viability and density in vitro. We cultured $\gamma \delta \mathrm{Tc}$ for $12-19$ days, then subjected them to $48 \mathrm{~h}$ in hypoxic $\left(2 \% \mathrm{O}_{2}\right)$ or normoxic $\left(20 \% \mathrm{O}_{2}\right)$ conditions. We found that exposure to hypoxia had variable effects on $\gamma \delta \mathrm{Tc}$ viability (Figure $2 \mathrm{~A}, P=0.08$ ), and significantly decreased cell density (Figure 2B, $P=5.7 \times 10^{-4}$ ). Immunophenotyping was performed using flow cytometric analyses of activation markers including $\gamma \delta$ TCR, NKG2D, CD56, CD69, CD95, CD40L, and HLA ABC as well as the inhibitory markers FasL and CD94. $\gamma \delta \mathrm{Tc}$ were stained with live/dead ZA prior to surface marker staining. Median fluorescence intensity values (MFIs) of hypoxia and normoxia samples were divided by the MFI of FMO controls to obtain fold-change values. Surface markers on $\gamma \delta$ Tc cultures subjected to 48 h 20 or $2 \% \mathrm{O}_{2}$ were not significantly different (Figures 2C-K).

\section{MIP1 $\alpha$, RANTES, and CD40L Are Secreted by $\gamma \delta \mathrm{Tc}$ in Hypoxia}

Culture supernatants from three different donor $\gamma \delta \mathrm{Tc}$ cultures subject to $40 \mathrm{~h}$ of normoxia or hypoxia were analyzed by cytokine array. While IL-8 appears elevated in the cumulative results graph depicted here (Figure 3A), this cytokine was only greatly increased under hypoxia in one of three experiments (Figure S2B in Supplementary Material, $P<0.0001$ ), was moderately increased in one experiment (Figure S2A in Supplementary Material, $P<0.05$ ), and not significantly elevated in the third experiment (Figure S2C in Supplementary Material). Due to significant variation among 


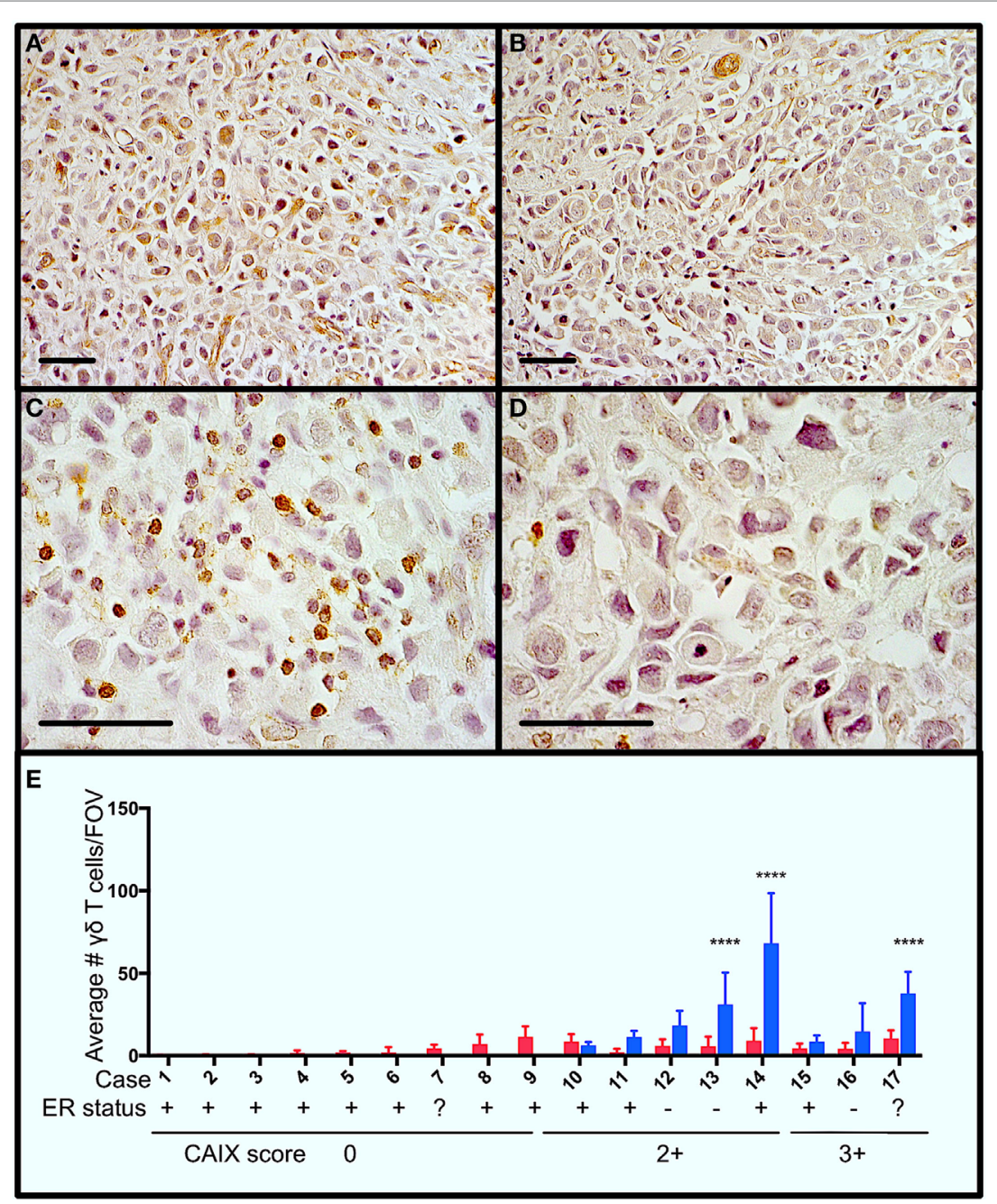

FIGURE 1 | Gamma delta T cells ( $\gamma \delta T c s)$ are present in areas of hypoxia in estrogen receptor positive (ER+) breast tumors. Serial sections from ER+ breast tumors were stained for carbonic anhydrase IX (CAIX) and T cell antigen receptor $\delta$. (A) Example of CAIX-positive staining at 400x magnification from case 14; (B) CAIX-negative field of view (FOV) from the same slide as in (A); (C) $\gamma \delta$ Tc in the same area as (A) at 1,000x magnification; and (D) $\gamma \delta \mathrm{Tc}$ in the same area as (B) at 1,000x magnification. Scale bars are $50 \mu \mathrm{m}$. Brown indicates positive staining. (E) Parallel staining for $\gamma \delta T c$ and CAIX suggests that $\gamma \delta T c$ infiltration increases in hypoxic regions. CAIX scoring is indicated below the case numbers: $0=$ no staining; $1=$ weak and/or very focal staining; $2+=$ strong but focal staining; and 3 = strong and extensive staining. Quantification and statistical analysis of $\gamma \delta$ Tc frequency in CAIX-positive versus -negative regions (blue and red bars, respectively) reveal significantly increased $\gamma \delta$ Tc infiltration in hypoxic regions (two-way ANOVA, ${ }^{* \star \star *} P<0.0001$ ).

donor cultures, cumulative results reveal significantly increased secretion of only CD40 ligand (CD40L or CD154) under hypoxia compared to normoxia (Figure 3A, $P=0.0472$ ). However, in all three individual cytokine arrays, significantly increased secretion of MIP $1 \alpha$ [or CCL3 = chemokine (C-C motif) ligand 3], RANTES (or CCL5), and CD40L under hypoxia compared to normoxia was observed (Figures S2A-C in Supplementary Material). Note that equal cell numbers were plated, and relative values at 2 and $1 \% \mathrm{O}_{2}$ were normalized to normoxia without taking harvested cell numbers into account. Considering the decrease in $\gamma \delta \mathrm{Tc}$ densities observed under hypoxia, this suggests an even greater effect would be observed if comparing the output of equal cell numbers.

ELISA validation for expression of RANTES, MIP1 $\alpha$, and CD40L was performed with culture supernatants from three different $\gamma \delta$ Tc cultures (Figures 3B-E, hypoxia $=1$ or $2 \% \mathrm{O}_{2}$ as indicated). For RANTES expression, an additional eight experiments were assayed, for secretion over $48 \mathrm{~h}$ at 20 or $2 \% \mathrm{O}_{2}$ (Figure 3C). In this case, and in contrast to the cytokine array data, ELISA values were normalized to cell numbers. Significantly increased secretion of these cytokines by $\gamma \delta \mathrm{Tc}$ was observed when cells were cultured in hypoxia compared to normoxia (asterisks indicate significance). A wide range of average secreted RANTES levels was observed, ranging from 93 to $521 \mathrm{pg} /$ million $\gamma \delta \mathrm{Tc}$ in normoxia to 431 to $856 \mathrm{pg} /$ million $\gamma \delta \mathrm{Tc}$ under hypoxia; the average ratio hypoxia:normoxia is indicated above the bars (Figures 3B-E). Likewise, secreted MIP1 $\alpha$ and CD40L levels were quantified for three independent experiments using ELISA (Figures 3D,E). MIP1 $\alpha$ levels ranged from 152 to $394 \mathrm{pg} / \mathrm{million}$ $\gamma \delta$ Tc in normoxia to 1,406 to $2,509 \mathrm{pg} /$ million $\gamma \delta$ Tc under hypoxia, 


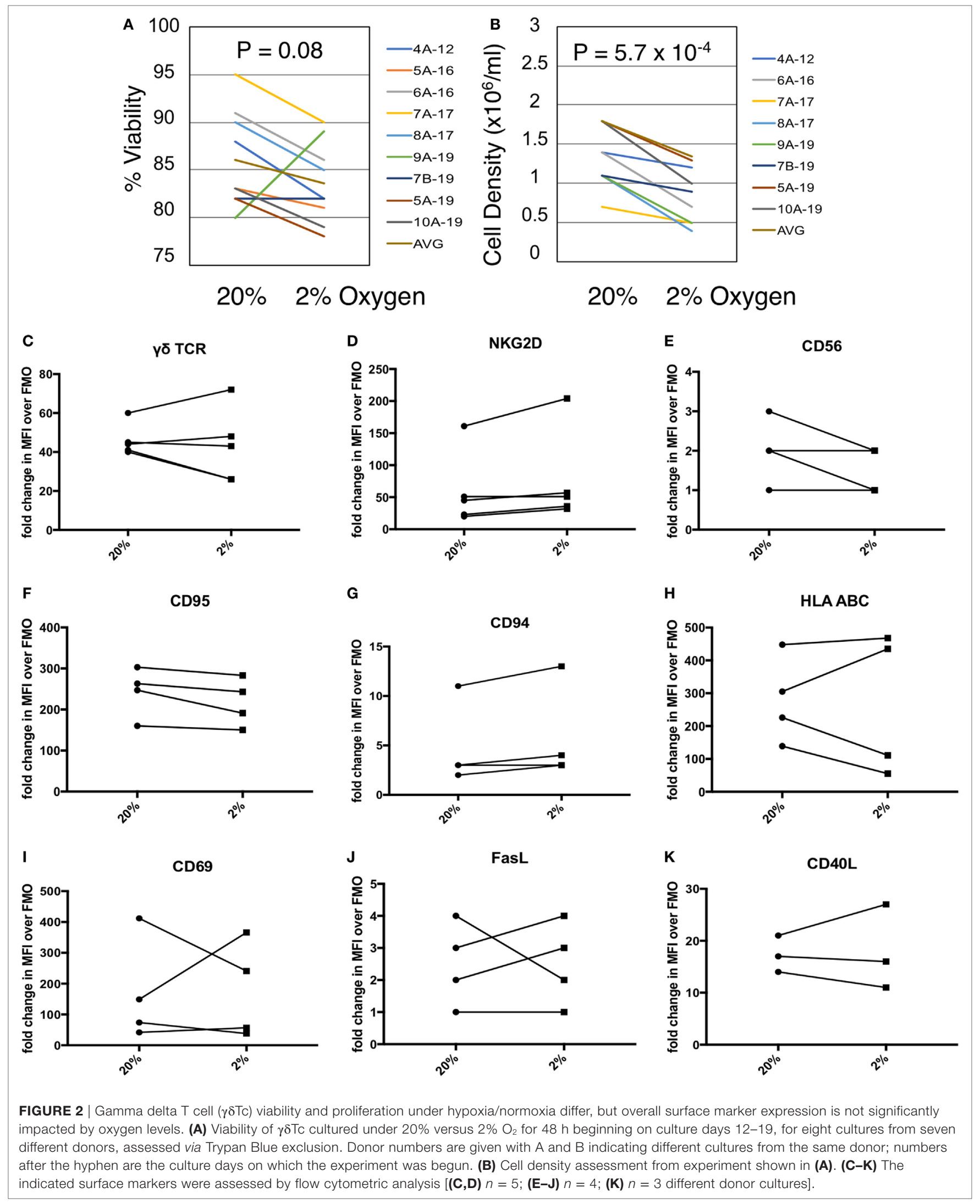


with fold changes from 4.0 to 14.2 (Figure 3D). Similarly, CD40L secretion by $\gamma \delta$ Tc increased significantly when cultured in low $\mathrm{O}_{2}$, with $2 \% \mathrm{O}_{2}$ in one experiment yielding an average of $171 \mathrm{pg}$ $\mathrm{CD} 40 \mathrm{~L} / \mathrm{million} \gamma \delta \mathrm{Tc}$ in hypoxia, a 4.9 -fold increase over just $35 \mathrm{pg}$
$\mathrm{CD} 40 \mathrm{~L} / \mathrm{million} \gamma \delta \mathrm{Tc}$ in normoxia (Figure 3E). Two experiments conducted with $1 \% \mathrm{O}_{2}$ yielded a wide range of CD40L secretion by $\gamma \delta \mathrm{Tc}$ in both conditions (Figure 3E, 120-395 and 536-653 pg $\mathrm{CD} 40 \mathrm{~L} / \mathrm{million} \gamma \delta \mathrm{Tc}$ in normoxia and hypoxia, respectively).
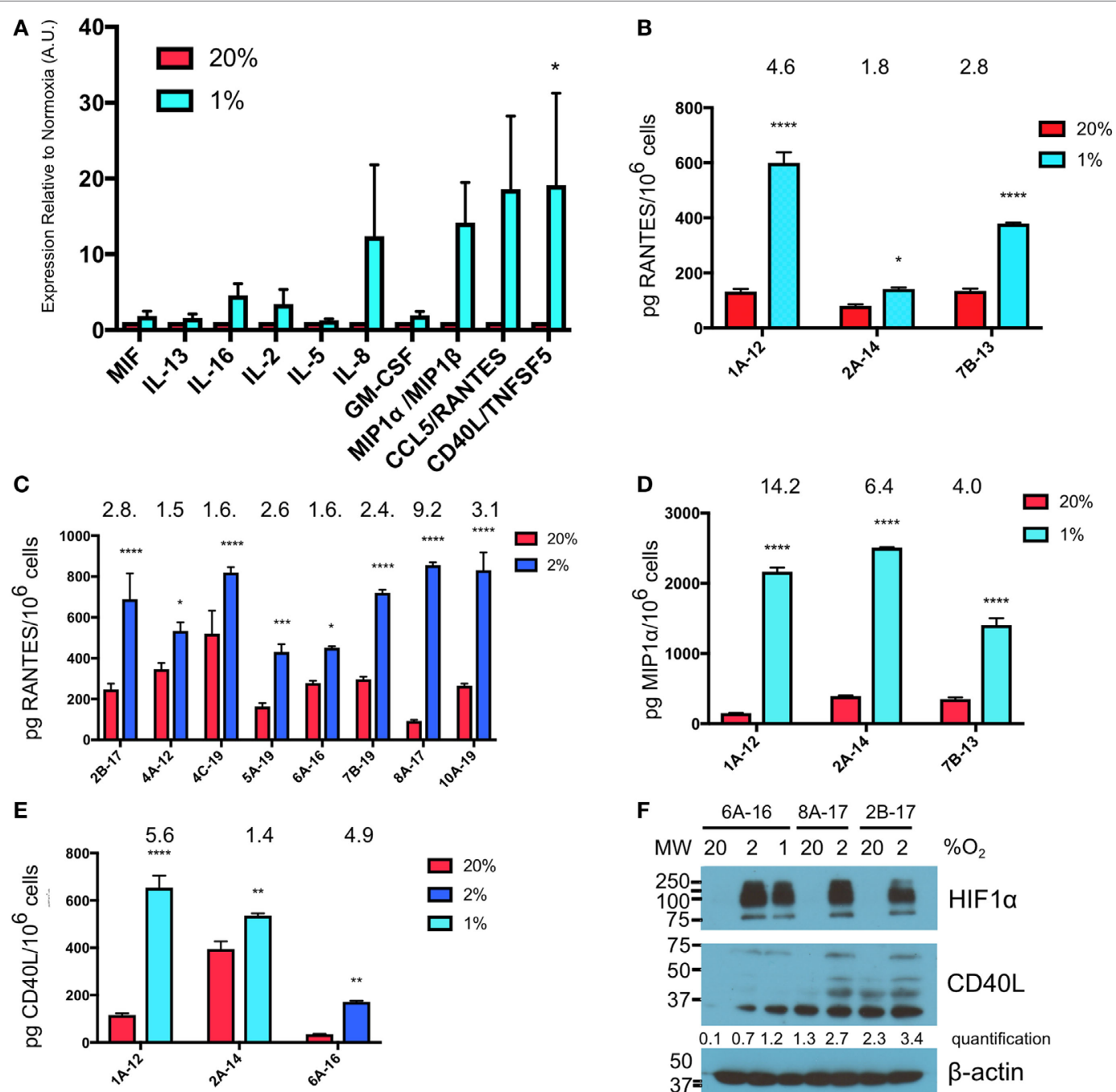

G
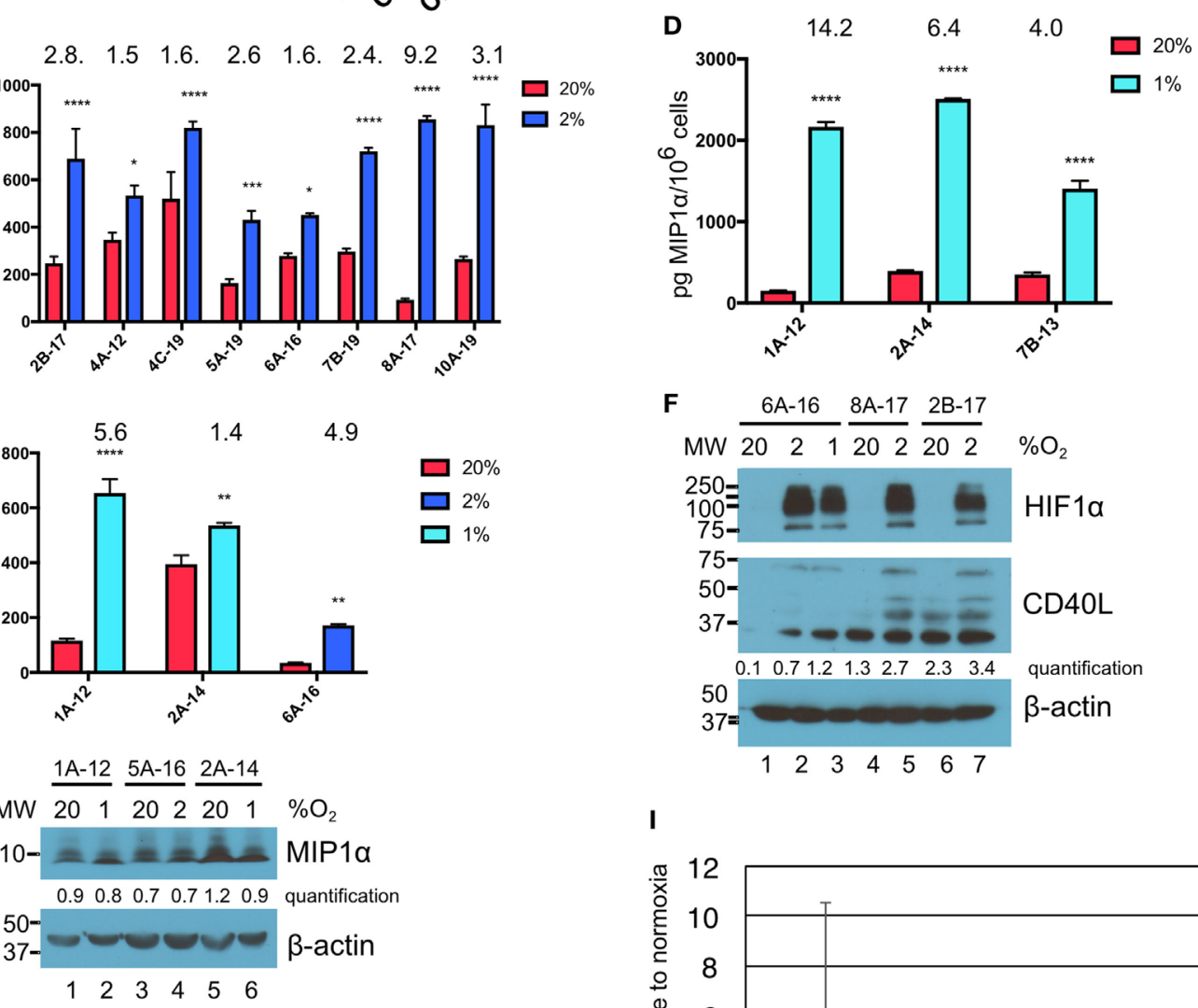

I

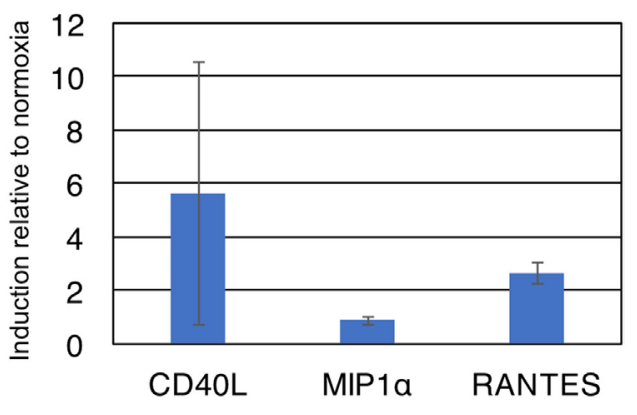

FIGURE 3 | Continued 
FIGURE 3 | Hypoxia induces secretion of macrophage inflammatory protein $1 \alpha$ (MIP1 $\alpha$ ), CCL5/regulated on activation, normal T cell expressed and secreted (RANTES), and CD40L/TNFSF5 by gamma delta T cells ( $\gamma \delta$ Tcs). (A) Culture supernatants from $\gamma \delta$ Tc subjected to $40 \mathrm{~h}$ at 20 or $1 \% \mathrm{O}_{2}$ were analyzed by cytokine array. Cumulative results of three independent experiments for a panel of cytokines that were differentially secreted by $\gamma \delta T c s$ under hypoxia compared to normoxia are shown. Error bars are SEM; A.U. = arbitrary units; (B) ELISA validation of RANTES cytokine results shown in (A) for three independent experiments carried out at 20 and $1 \% \mathrm{O}_{2}$ for $40 \mathrm{~h}$; (C) RANTES ELISA for eight hypoxia experiments carried out for $48 \mathrm{~h}$ at 20 and $2 \%$ O ; (D) MIP1 $\alpha$ ELISA for the same experiments shown in (B); (E) CD40L ELISA for culture 6A-16 subject to $48 \mathrm{~h} 20$ or $2 \% \mathrm{O}_{2}$, and two of the experiments shown in (B,D). Statistical analyses for (A-E): two-way ANOVA, ${ }^{\star} P<0.05,{ }^{\star \star} P<0.01,{ }^{\star \star \star} P<0.001$, ${ }^{\star \star \star \star} P<0.0001$; (F-H) Western blot analysis of lysates from $\gamma \delta$ Tc cultures subject to 20,2 , and/or $1 \%$ O for $48 \mathrm{~h}$ as indicated. $\gamma \delta$ Tc culture identification is given above the blots and molecular weight (MW) markers are shown on the left; corresponding $\beta$-actin loading controls are shown in the bottom panels; relative band intensities were quantified and are indicated in arbitrary units; (F) three examples shown for detection of hypoxia inducible factor 1-alpha (HIF1 $\alpha)(n=6,5$ different donors) and CD40L ( $n=8,7 \gamma \delta$ Tc cultures from six donors); (G) MIP1 $\alpha(n=7,6 \gamma \delta$ Tc cultures from five donors); (H) RANTES $(n=7)$; and $(\mathbf{I})$ induction of proteins in $(\mathbf{F}-\mathbf{H})$ was determined by dividing protein band intensities from hypoxic samples by their corresponding normoxia control, and averaging these values. Error bars are SD.

Western blotting was done to verify induction of HIF1 $\alpha$ in $\gamma \delta \mathrm{Tc}$ under hypoxia, and also to investigate whether intracellular levels of CD40L, MIP1 $\alpha$, and RANTES reflected those of secreted proteins (Figures 3F,G). HIF $1 \alpha$ was clearly induced in $\gamma \delta \mathrm{Tc}$ at 2 and $1 \% \mathrm{O}_{2}$ in all cases; three examples from six independent experiments with five donor cultures are shown (Figure 3F, top panel, compare lane 1 versus 2 and 3, 4 versus 5, and 6 versus 7). CD40L appears visibly increased in hypoxia samples for $\gamma \delta \mathrm{Tc}$ culture $6 \mathrm{~A}-16$ (Figure $3 \mathrm{~F}$, middle panel, compare lane 1 versus 2 and 3), and quantification suggests this is also the case for the other two donor cultures shown (lane 4 versus 5 and lane 6 versus 7). Note that several forms of CD40L are evident here, which were included in the quantification of bands. Of eight experiments with seven $\gamma \delta \mathrm{Tc}$ cultures from six donors, intracellular CD40L was clearly visibly increased in three (38\%). HIF1 $\alpha$ and CD40L blots originated from the same gel, which was transferred and then cut at $75 \mathrm{kDa}$; thus, the $\beta$-actin loading control serves for both (Figure 3F, lower panel). MIP1 $\alpha$ levels were not consistently higher in $\gamma \delta$ Tc subject to hypoxia versus normoxia (Figure 3G, representative of seven experiments with six $\gamma \delta T c$ cultures from five donors), as demonstrated by very similar quantification values within each experiment. By contrast, RANTES was typically induced by hypoxia, with higher protein levels evident in cellular lysates from $\gamma \delta \mathrm{Tc}$ cultured in 1 or $2 \% \mathrm{O}_{2}$ compared to normoxia (Figure $3 \mathbf{H}$, compare lane 1 versus 2 and 3, 4 versus 5, and 8 versus 9; $n=$ seven independent experiments, seven donors, induction clear in six, unclear in one). Longer exposure of this blot also revealed RANTES induction in lane 7 versus 6 (Figure S3 in Supplementary Material). Full scans of Western blots can be found in Figure S4 in Supplementary Material. The average induction of CD $40 \mathrm{~L}, \mathrm{MIP} 1 \alpha$, and RANTES in $\gamma \delta \mathrm{Tc}$ under hypoxia relative to normoxia was calculated using Western blot band intensity values, and confirmed elevated levels of intracellular CD40L and RANTES, but not MIP1 $\alpha$, under hypoxia (Figure 3I).

\section{NKG2D Expressed on $\gamma \delta$ Tc and MICA/B on Breast Cancer Targets Are Critical for $\gamma \delta \mathrm{TC}$ Killing}

MCF-7 and T47D are estrogen receptor (ER) positive luminal A breast carcinoma cell lines (35). Both of these cell lines express $\mathrm{MICA} / \mathrm{B}$ on the surface as identified by flow cytometric analysis (Figures 4A,B). Blocking NKG2D on $\gamma \delta$ Tc significantly decreased lysis of MCF-7 (Figure 4C, one-way ANOVA versus IgG control,
$P<0.0001$, representative of four independent experiments, $n=4)$ and T47D (Figure 4D, $P=0.0002, n=5$ ). Likewise, blocking the NKG2D ligand MICA/B on targets prevented MCF-7 and T47D cell lysis (Figures 4C,D, both $P<0.0001$, $n=2$ and 3 , respectively). By contrast, no decrease in cell lysis of either line was observed when $\gamma \delta T c$ were pre-incubated with antibodies against MIP1 $\alpha$, RANTES, or CD40L (Figures 4E,F, $n=3$ and 2 , respectively). Since antibodies were not washed away prior to co-incubation with targets, blocking should have been effective against both membrane-bound and soluble proteins. Thus, it appears that MIP1 $\alpha$, RANTES, and CD40L are not directly involved in $\gamma \delta \mathrm{Tc}$ cytotoxicity against MCF-7 or T47D.

\section{$\gamma \delta$ Tc Cytotoxicity Against MCF-7 and T47D Targets Is Enhanced in Hypoxia}

Cytotoxicity experiments were performed in which $\gamma \delta \mathrm{Tc}$ effectors and breast cancer cell lines were pre-incubated for $48 \mathrm{~h}$ under normoxia or hypoxia $\left(2 \% \mathrm{O}_{2}\right)$ and then co-cultured at 1:1, 10:1, and 20:1 E:T ratios in parallel under normoxia or hypoxia, as per target pre-incubation conditions, for $4 \mathrm{~h}$. Pre-incubation in hypoxia enhanced $\gamma \delta$ Tc cytotoxicity against MCF-7 targets cultured in normoxia (Figures 5A,B). In a representative example, significantly increased MCF-7 cell lysis was observed at 20:1 (Figure 5A, $P=0.0005$ ); when data from all six experiments performed with day $21 \gamma \delta \mathrm{Tc}$ from five different donors (six different cultures) were compiled and subject to statistical analysis, this result was confirmed (Figure 5B, $P=0.007$ ). Likewise, $\gamma \delta \mathrm{Tc}$ cultured in hypoxia were better able to kill T47D cultured in normoxia (Figures 5C-D). In an example representative of five experiments with day $21 \gamma \delta \mathrm{Tc}$ from four different donors, target cell lysis was significantly increased at all E:T ratios tested (Figure 5C, $P<0.01$ ); analysis of compiled results from all five experiments revealed significantly increased lysis of targets by hypoxia-treated $\gamma \delta \mathrm{Tc}$ at 1:1 and 20:1 E:T (Figure 5D, $P<0.05$ ).

\section{Breast Cancer Targets in Hypoxia Are Resistant to $\gamma \delta$ Tc Killing due to MICA Shedding}

As outlined above, cytotoxicity experiments were performed in which breast cancer cell lines were pre-incubated for $48 \mathrm{~h}$ under normoxia or hypoxia $\left(2 \% \mathrm{O}_{2}\right)$ and then co-cultured with $\gamma \delta \mathrm{Tc}$ at $1: 1,10: 1$, and 20:1 E:T in parallel under normoxia or hypoxia for 


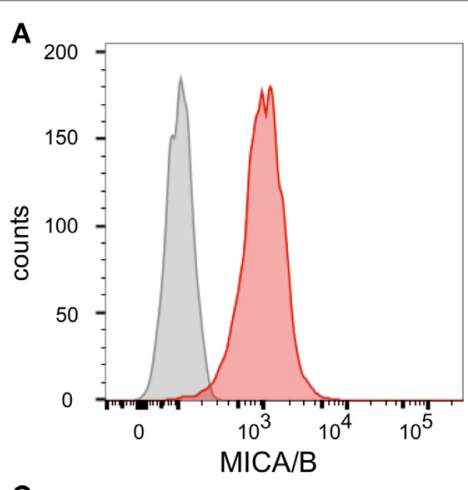

C

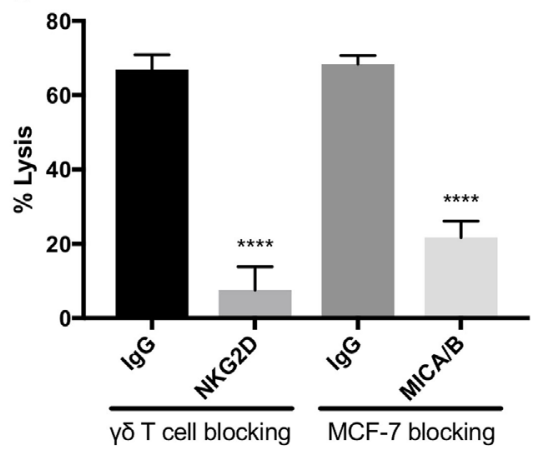

E

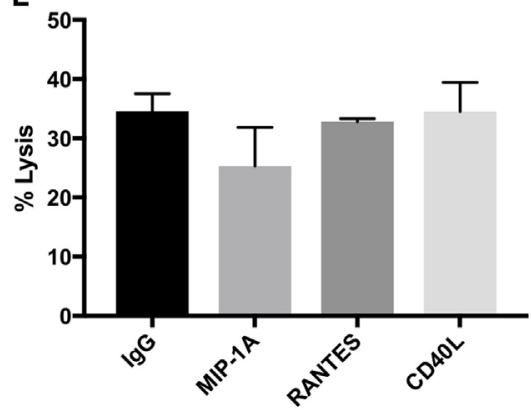

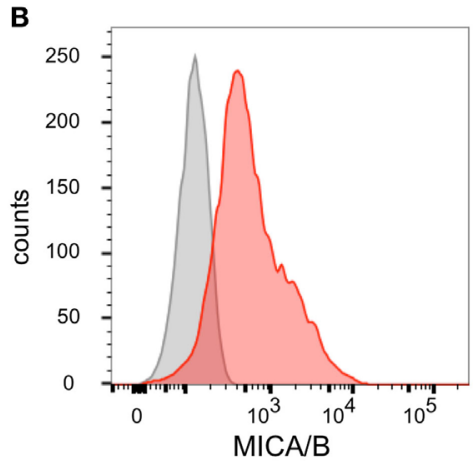

D

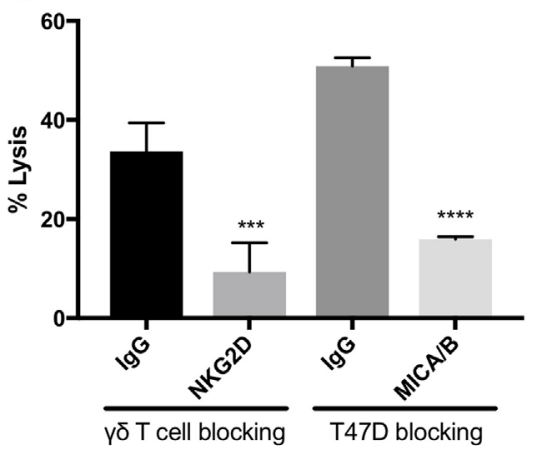

$\mathbf{F}$

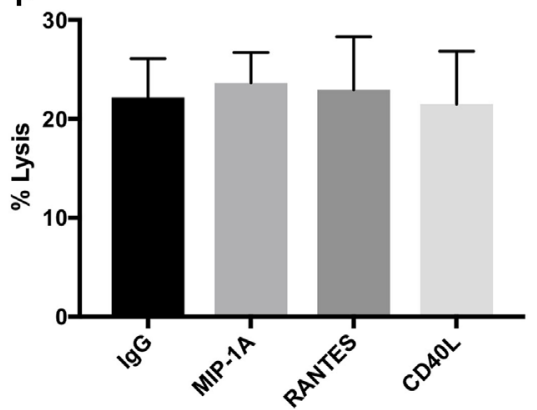

FIGURE 4 | Natural killer group 2, member D (NKG2D) on gamma delta T cells ( $\gamma \delta$ Tcs) and MHC class I polypeptide-related sequence A (MICA)/B on breast cancer cell lines mediate $\gamma \delta$ TC cytotoxicity. Flow cytometric analysis of $\mathbf{( A ) ~ M C F - 7 ~}(n=4)$ and $\mathbf{( B )}$ T47D $(n=2)$ confirms that both cell lines express MICA/B. (C) Cytotoxicity assays in which NKG2D on $\gamma \delta$ Tcs or MICA/B on MCF-7 cells are blocked with antibodies confirm $\gamma \delta$ Tc recognition of breast cancer targets via this receptor/ligand interaction ( $n=3$, representative of three independent experiments with three different donor cultures). (D) Blocking assays as in (C) using T47D targets ( $n=3$ ). (E) Blocking macrophage inflammatory protein $1 \alpha(\mathrm{MIP1} \alpha)$, CCL5/regulated on activation, normal T cell expressed and secreted (RANTES), and CD40L/TNFSF5 does not decrease lysis of MCF-7 $\left(n=3\right.$ independent experiments with two different donor cultures) or (F) T47D $(n=2)$. Statistical analyses for $(\mathbf{C}-\mathbf{F})$ : one-way ANOVA, ${ }^{\star \star \star} P<0.001$, ${ }^{\star \star \star \star} P<0.0001$.

$4 \mathrm{~h}$. In most cases $(4 / 6,67 \%)$, pre-incubation in hypoxia induced MCF-7 resistance to $\gamma \delta$ Tc cytotoxicity (Figures 6A-C). In a representative example from an experiment performed with $\gamma \delta T c$ culture 4B-21, significantly decreased MCF-7 cell lysis was observed at 10:1 (Figure 6A, $P=0.0054$ ) and 20:1 (Figure 6A, $P=0.0119$ ). By contrast, in two experiments with two different $\gamma \delta$ Tc cultures from the same donor, no resistance was observed; one example is shown in which MCF-7 cultured under hypoxia appeared to be more susceptible to $\gamma \delta$ Tc killing (Figure 6B, $P<0.0001$ at 1:1 and 10:1). When data from five experiments performed with day $21 \gamma \delta \mathrm{Tc}$ from five different donors were compiled and subject to statistical analysis, the overall effect of hypoxia inducing MCF-7 resistance was confirmed (Figure 6C, $P=0.0011$ ). Likewise, T47D cultured in hypoxia were more resistant to $\gamma \delta$ Tc killing at
20:1 than those cultured in normoxia (Figure 6D, $P=0.0043$ ), although the $1: 1$ result is opposite $(P=0.0076)$; these compiled results were from four experiments conducted with four different $\gamma \delta \mathrm{Tc}$ donor cultures. Flow cytometric analysis of MICA/B surface expression on breast cancer lines subjected to $48 \mathrm{~h}$ normoxia or hypoxia revealed no significant change in MFI; representative examples are shown for MCF-7 (Figure 6E, $n=4$ ) and T47D (Figure 6F, $n=2$ ). Of note, Accutase was used for dissociation of these adherent cell lines, out of concern for potential trypsin sensitivity of surface MICA/B that might have confounded our results. Supernatants from MCF-7 and T47D subject to $48 \mathrm{~h}$ 20 or $2 \% \mathrm{O}_{2}$ were subject to MICA ELISA (Figure 6G). MICA could not be detected in supernatants directly, thus samples were concentrated and MICA ELISA was repeated. MICA in T47D 

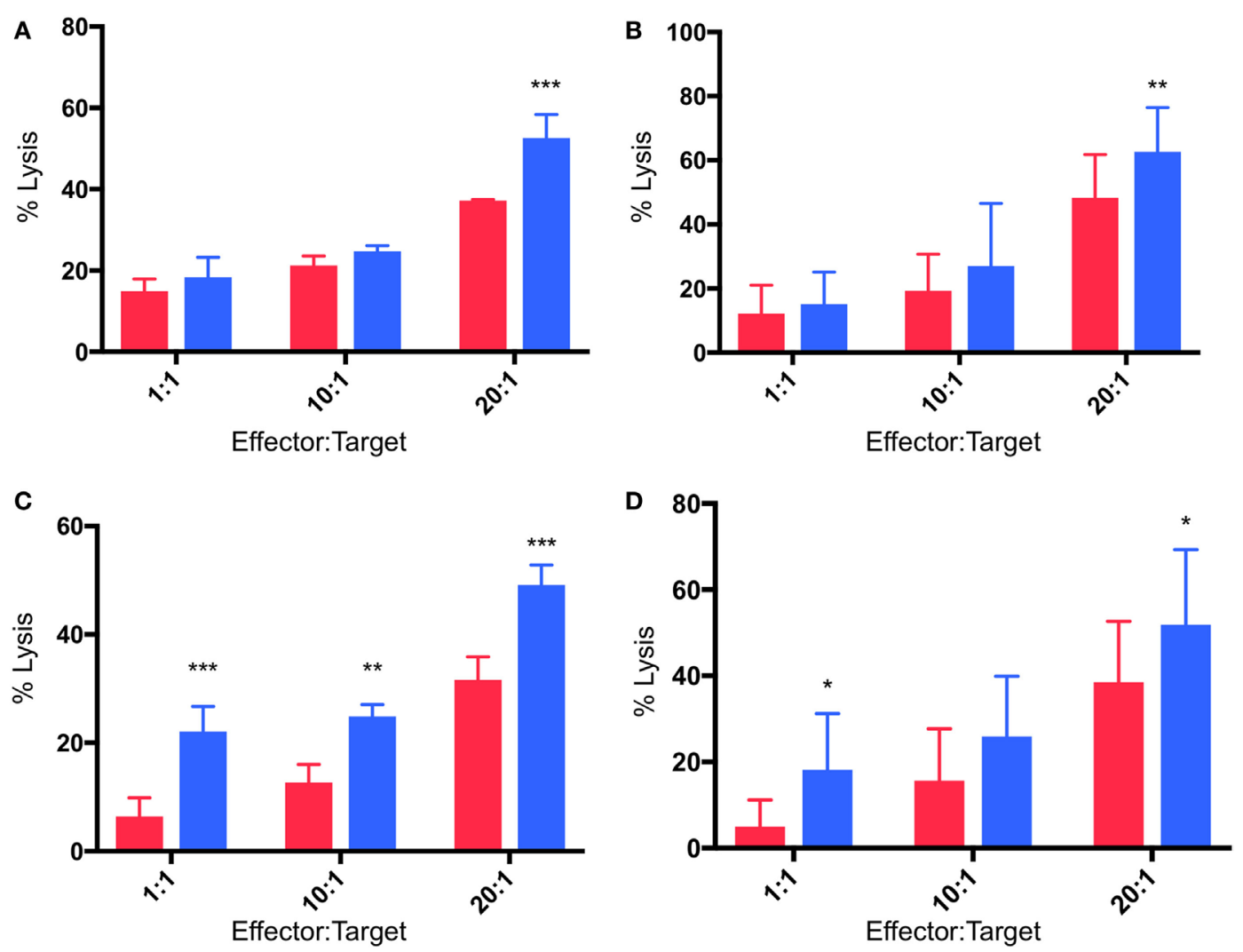

FIGURE 5 | Enhanced cytotoxicity of gamma delta T cells $\left(\gamma \delta\right.$ Tcs) cultured in hypoxia. Cytotoxicity assays comparing $\gamma \delta T c$ cultured in $20 \%$ (red bars) or $2 \% \mathrm{O}_{2}$ (blue bars) $48 \mathrm{~h}$ prior to co-culture with breast cancer target lines cultured at $20 \% \mathrm{O}_{2}$. (A) A representative example of $\gamma \delta T c$ targeting MCF-7 cells; (B) compiled results from six independent experiments with $\gamma \delta$ Tc cultures from five different donors targeting MCF-7; (C) a representative example with T47D targets; (D) compiled results from five independent experiments with $\gamma \delta$ Tc cultures from four different donors targeting T47D. Two-way ANOVA, ${ }^{\star} P<0.05$, ${ }^{\star \star} P<0.01$, ${ }^{\star \star \star} P<0.001$.

remained below the detection limit; however, after normalization to cell numbers, a significant increase in secreted MICA by MCF-7 cells under hypoxia was observed in $3 / 4$ experiments (Figure 6G, $\left.{ }^{* * *} P=0.0005,{ }^{* * *} P<0.0001\right)$. These results match those observed in cytotoxicity experiments, with ELISA from MCF-7 targets used in cytotoxicity assays with 4B-21 showing increased MICA secretion under hypoxia that fits with the observed resistance to $\gamma \delta \mathrm{Tc}$ cytotoxicity in Figure 6A. Likewise, no difference in MICA secretion was observed in MCF-7 targets under 20 or $2 \% \mathrm{O}_{2}$ subject to cytotoxicity assays with $\gamma \delta \mathrm{Tc}$ culture $10 \mathrm{~B}-21$, which also showed no MCF-7 resistance to $\gamma \delta$ Tc killing in Figure 6B. Thus, resistance to $\gamma \delta$ Tc killing appears to be correlated with MICA secretion by breast cancer targets. Despite enhanced cytotoxicity of $\gamma \delta \mathrm{Tc}$ cultured under $2 \%$ compared to $20 \% \mathrm{O}_{2}$ against targets cultured under normoxia (Figure 5), they are unable to overcome resistance exhibited by MCF-7 under $2 \% \mathrm{O}_{2}$, as revealed by analysis of five compiled experiments comparing $\gamma \delta \mathrm{Tc}$ cultured under 20 or $2 \% \mathrm{O}_{2}$ against MCF-7 cells cultured in hypoxia (Figure $6 \mathbf{H}$ ).

\section{DISCUSSION}

Gamma delta $\mathrm{T}$ cells are being developed as immunotherapeutic agents for a variety of cancer indications and clinical trials (Phase I/II) thus far have shown excellent safety profiles (36). Yet, they are known to embody remarkable functional plasticity, dependent on the environment in which they find themselves $(24,37-39)$. Thus, it is important to explore the function of $\gamma \delta \mathrm{Tc}$ infiltrating solid tumors, some of which may be hypoxic. In our small cohort of 17 breast cancer cases, $47 \%$ of tumors contained areas of CAIX positivity indicating hypoxia (Figure 1). The CAIX-negative cases were 89\% ER+ (Figure 1E, cases $1-9$, case 7 was of unknown ER status); of ER+ cases, $76 \%$ were CAIX negative. This confirms reports showing up to 80\% CAIX negativity in studies assessing ER+ breast tumors; in these cases, CAIX negativity correlated with low histological grade (40). While our cohort was admittedly small, the very low levels of $\gamma \delta \mathrm{Tc}$ infiltrates in CAIX-negative tumors, correlated with low histological grade, confirm results showing that levels of $\gamma \delta \mathrm{Tc}$ infiltration correlate positively with higher histological grades (41). Unfortunately, our cohort size was too limited to determine whether $\gamma \delta$ Tc infiltration correlated with patient outcome. We did, however, find $\gamma \delta \mathrm{Tc}$ in areas of hypoxia in some tumors. While we did not have the power in our study, or in vivo functional data, to claim that $\gamma \delta \mathrm{Tc}$ are preferentially attracted to hypoxic regions, our results at least provide an indication that $\gamma \delta \mathrm{Tc}$ can be found in hypoxic areas of tumors, and that studying 
their function under low $\mathrm{O}_{2}$ is worthwhile. As CAIX is associated more so with triple negative breast cancers (TNBC) $(18,42)$, future studies of $\gamma \delta T c$ and hypoxia should focus on a larger cohort of TNBC patients. Indeed, the groundwork for such studies has been laid by Hidalgo and colleagues, who recently reported on the pattern of distribution of $\gamma \delta \mathrm{Tc}$ in TNBC (43).
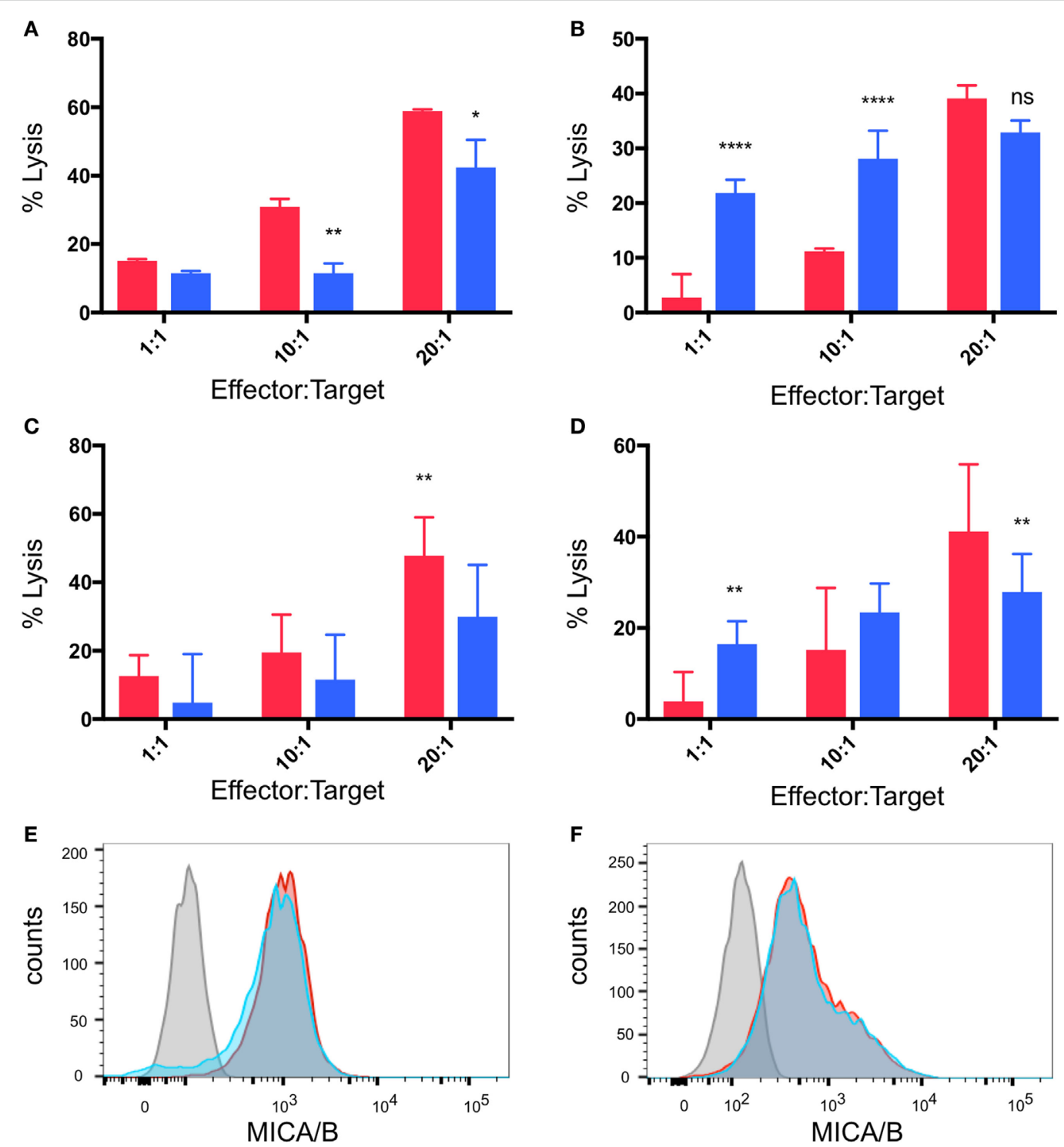

$\mathbf{F}$
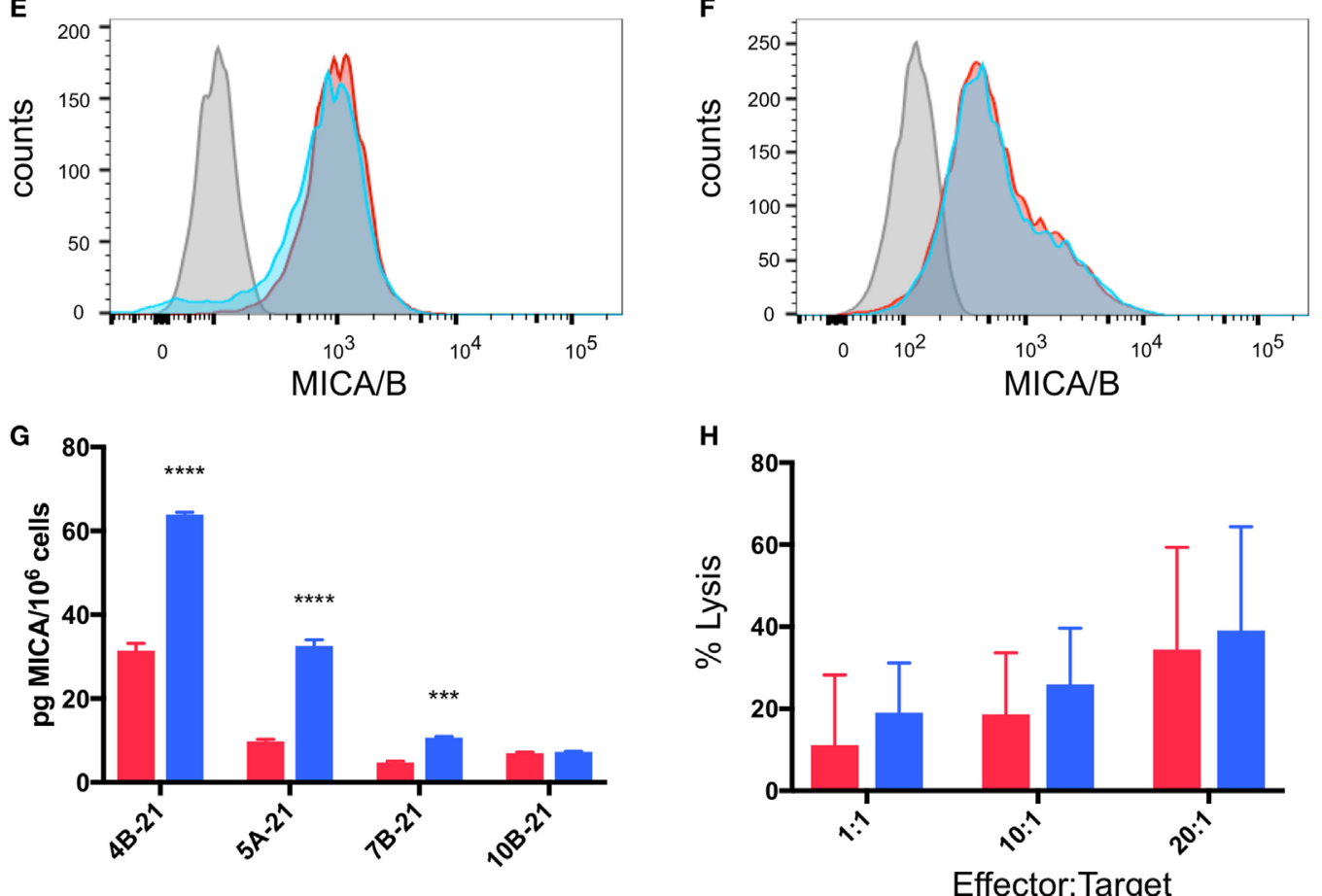


\begin{abstract}
FIGURE 6 | Breast cancer cell lines pre-incubated in hypoxia are resistant to gamma delta T cell ( $\gamma \delta$ Tc) killing. Cytotoxicity assays comparing the ability of $\gamma \delta T c$ cultured under normoxia to target breast cancer target lines cultured at $20 \% \mathrm{O}_{2}$ (red bars) or $2 \% \mathrm{O}_{2}$ (blue bars) for 48 h prior to co-culture under hypoxia; (A) a representative example in which MCF-7 cells were resistant to $\gamma \delta$ Tc killing (4B-21); (B) an example in which MCF-7 cells cultured under $2 \% \mathrm{O}_{2}$ were susceptible to $\gamma \delta$ Tc killing (10B-21); (C) compiled results from five independent experiments with $\gamma \delta$ Tc cultures from five different donors targeting MCF-7; (D) compiled results from four experiments with four different donor-derived $\gamma \delta$ Tc cultures targeting T47D; (E) surface expression of MHC class I polypeptiderelated sequence $\mathrm{A}$ (MICA)/B on T47D remains unchanged under hypoxia versus normoxia; (F) surface expression of MICA/B on MCF-7 is not differentially impacted by hypoxia versus normoxia; (G) MICA ELISA on concentrated supernatants of MCF-7 from experiments in (A); (H) compiled results from five independent experiments with $\gamma \delta$ Tc cultures from five different donors cultured at $20 \% \mathrm{O}_{2}$ or $2 \% \mathrm{O}_{2}$ targeting MCF-7 cultured under hypoxia for $48 \mathrm{~h}$ prior to co-culture under hypoxia. Two-way ANOVA, ${ }^{\star} P<0.05,{ }^{\star \star} P<0.01,{ }^{\star \star \star} P<0.001,{ }^{\star \star \star \star} P<0.0001$.
\end{abstract}

It was unsurprising that $\gamma \delta$ Tc cell density decreased under hypoxia (Figure 2), as terminally differentiated $\gamma \delta \mathrm{Tc}$ stop proliferating to become cytotoxic (44), and hypoxia enhanced $\gamma \delta \mathrm{Tc}$ cytotoxicity (Figure 5). Delayed cell-cycle progression was also noted in a study on PBMC in hypoxia (45). To our knowledge, the only study of $\gamma \delta \mathrm{Tc}$ in the context of hypoxia showed that circulating $\gamma \delta \mathrm{Tc}$ in patients with obstructive sleep apnea had elevated intracellular tumor necrosis factor alpha (TNF $\alpha)$ and IL-8 levels, increased TNF $\alpha$ and L-selectin-mediated adhesion properties, and enhanced cytotoxicity against endothelial cells compared to those isolated from healthy donors (46). While that study compared freshly isolated blood-derived $\gamma \delta \mathrm{Tc}$ from patients and healthy donors, we used healthy donor-derived in vitro expanded $\gamma \delta \mathrm{Tc}$ for our experiments, which potentially accounts for different results. TNF $\alpha$ secretion was not impacted by hypoxia in our study, as no differential effects were detected by cytokine array (data not shown). While we did observe strongly elevated hypoxia-induced IL-8 in the supernatant of one of the three $\gamma \delta$ Tc cultures subject to cytokine array analysis (Figure S2 in Supplementary Material), this was not the case for the other two cultures.

More significant were cytokine array data pointing to increased secretion of RANTES, MIP $1 \alpha$, and CD40L by $\gamma \delta T c$ under low $\mathrm{O}_{2}$ compared to normoxia that were confirmed by subsequent ELISAs (Figures S2A-C in Supplementary Material; Figures 3B-E). Intracellular protein levels induced by hypoxia matched ELISA results only in the case of RANTES (Figure 3H); the same could not be said for CD40L and MIP1 $\alpha$, where hypoxia treatment did not appear to increase intracellular levels (Figures 3F,G), and surface expression of CD40L was variable (Figure 2K). Since blocking these proteins appeared to have no impact on $\gamma \delta \mathrm{Tc}$ cytotoxicity against breast cancer target lines (Figures 4E,F), they must have an indirect function related to enhanced cytotoxicity of $\gamma \delta$ Tc under hypoxia.

Human memory $\mathrm{V} \gamma 2 \mathrm{~V} \delta 2$ cells were reported to store cytoplasmic RANTES that was secreted rapidly in response to TCR signaling, but little MIP1 $\alpha$ protein was found in these cells (47). RANTES is a chemokine employed to recruit antigen presenting cells, such as dendritic cells $(48,49)$, and thus speaks to the anti-tumor function of $\gamma \delta \mathrm{Tc}$ in hypoxia, though breast tumors may use this to their own advantage to promote malignancy (50). RANTES and MIP1 $\alpha$ expression were also reported to aid V $\delta 1$ cell suppression of HIV replication (51). CD40 ligation is thought to enhance the immunogenicity of tumors (52), thus $\gamma \delta \mathrm{Tc}$ may secrete CD40L in order to better "see" tumor targets. CD40L may also inhibit growth of CD40-expressing tumors directly (52-55). Further investigation will be required to determine the functions served by these cytokines with respect to $\gamma \delta T c$ targeting solid tumors.

A study of the $\mathrm{V} \gamma 9 \mathrm{~V} \delta 2 \gamma \delta \mathrm{Tc}$ subset in the context of breast cancer suggested that surface levels of MICA/B on breast cancer target cell lines were associated with $\gamma \delta \mathrm{Tc}$ cytotoxicity against these lines; however, direct blocking assays were not carried out (16). Both MCF-7 and T47D cells expressed surface MICA/B, in contrast to an earlier report suggesting a lack of MICA/B expression on MCF-7 (56). If trypsin was used to dissociate MCF-7 in that study, it might explain their inability to detect MICA/B; to avoid this issue, we used Accutase to dissociate our adherent cell lines, as detachment of cells is gentler and protects most surface epitopes. We have confirmed the involvement of NKG2D on $\gamma \delta \mathrm{Tc}$ and MICA/B on MCF-7 and T47D in cytotoxicity of $\gamma \delta$ Tc against breast tumor targets (Figure 4), although differences in the ability of $\gamma \delta \mathrm{Tc}$ to kill targets pre-incubated in hypoxia or normoxia do not appear to be related to surface levels of MICA (Figure 6).

One mechanism of hypoxia-mediated tumor evasion is MICA shedding (57). MICA downregulation related to shedding under hypoxia, as well as downregulated expression of NKG2D on PBMCs incubated with culture supernatants of prostate cancer cells exposed to hypoxia-abrogated upon incubation with MICA blocking antibodies-has been reported (58). MICA shedding is not a universal evasion mechanism employed by all cancer cells, however, as glioblastoma cell lines did not shed MICA, although this study was only carried out under normoxia (59). While we assume that soluble MICA may bind NKG2D and block or downregulate this receptor to prevent $\gamma \delta$ Tc recognition of breast cancer targets, a recent report suggests that, in mice, soluble NKG2D might activate NK cells and aid in tumor eradication, but this anti-tumor effect has yet to be shown in humans or with $\gamma \delta \mathrm{Tc}(60)$. By contrast, soluble MIC was shown to decrease $\gamma \delta \mathrm{Tc}$ cytotoxicity in pancreatic cancer (61) and has been implicated in evasion of human ovarian cancer cells from $\gamma \delta \mathrm{Tc}$ recognition (21). Thus, we were surprised that surface expression of MICA/B on MCF-7 and T47D breast cancer lines appeared unaffected by $48 \mathrm{~h}$ under hypoxia (Figure 6). However, MICA secretion did not correlate with MICA surface levels, as soluble MICA increased in the supernatants of MCF-7 cells cultured under hypoxia, while surface MICA levels remained unchanged (Figure 6). Thus, it appears that we would need to neutralize soluble MICA to improve $\gamma \delta$ Tc cytotoxicity, since target surface expression did not appear to be affected by hypoxia. That said, we did not directly assess MICA expression during co-culture with $\gamma \delta \mathrm{Tc}$, and it is possible that MICA was downregulated in the presence of $\gamma \delta \mathrm{Tc}$, although the correlation between resistance to $\gamma \delta$ Tc killing and soluble MICA levels in culture supernatants under hypoxia 
speaks against this (Figure 6). One way to overcome MICA shedding may be to increase nitric oxide signaling (58), although its impact on $\gamma \delta$ Tc would have to be assessed.

Although the $\gamma \delta \mathrm{Tc}$ tumor infiltrating lymphocytes (TIL) signature was deemed the most positive prognosticator across a range of cancers, including breast cancer (62), some reports suggest that $\gamma \delta \mathrm{Tc}$ may take on a regulatory phenotype within the breast TME $(41,56,63,64)$. In one study, $\gamma \delta T c$ TIL isolated from a breast tumor were expanded in high levels of IL-2 for several weeks prior to immunosuppression assays and proved to inhibit dendritic cell maturation and CD8+ T cell cytotoxicity (56); however, given the known functional plasticity of $\gamma \delta \mathrm{Tc}$, such assays conducted on ex vivo expanded cells removed from the TME cannot inform the function of $\gamma \delta \mathrm{Tc}$ in situ. A positive correlation was observed between $\gamma \delta \mathrm{Tc}$ infiltration and breast cancer stage, leading the authors to suggest that $\gamma \delta \mathrm{Tc}$ may contribute to disease pathology; however, causality was not established (41). Although our cohort size was much smaller, we too observed a positive correlation between CAIX expression, indicating hypoxia-typically an indicator of cancer progression-and $\gamma \delta$ Tc infiltration (Figure 1). This could just as easily indicate the greater need for $\gamma \delta \mathrm{Tc}$ attempting to eradicate disease. Our hypoxia experiments reveal enhanced cytotoxicity of $\gamma \delta \mathrm{Tc}$ exposed to $48 \mathrm{~h}$ of low $\mathrm{O}_{2}$, suggesting that $\gamma \delta \mathrm{Tc}$ are indeed able to kill in this environment (Figure 5). Soluble MICA appears to inhibit $\gamma \delta$ Tc cytotoxicity against breast tumor targets in hypoxia and, despite their increased killing capacity under low $\mathrm{O}_{2}, \gamma \delta \mathrm{Tc}$ are unable to overcome resistance exhibited by MCF-7 under $2 \% \mathrm{O}_{2}$ (Figure 6), a condition under which $\gamma \delta \mathrm{Tc}$ must operate within at least some parts of a tumor. Further studies will be required to definitively identify $\gamma \delta$ Tc function in breast tumors in situ.

\section{ETHICS STATEMENT}

This study was carried out in accordance with the recommendations of the Research Ethics Guidelines, Health Research Ethics Board of Alberta-Cancer Committee with written informed consent from all subjects. All subjects gave written informed consent in accordance with the Declaration of Helsinki.

\section{REFERENCES}

1. Vaupel P, Hockel M, Mayer A. Detection and characterization of tumor hypoxia using pO2 histography. Antioxid Redox Signal (2007) 9:1221-35. doi:10.1089/ars.2007.1628

2. Le QT, Denko NC, Giaccia AJ. Hypoxic gene expression and metastasis. Cancer Metastasis Rev (2004) 23:293-310. doi:10.1023/B:CANC.0000031768. 89246.d7

3. Postovit LM, Adams MA, Lash GE, Heaton JP, Graham CH. Oxygen-mediated regulation of tumor cell invasiveness. Involvement of a nitric oxide signaling pathway. J Biol Chem (2002) 277:35730-7. doi:10.1074/jbc.M204529200

4. Subarsky P, Hill RP. The hypoxic tumour microenvironment and metastatic progression. Clin Exp Metastasis (2003) 20:237-50. doi:10.1023/ A:1022939318102

5. Brizel DM, Scully SP, Harrelson JM, Layfield LJ, Bean JM, Prosnitz LR, et al. Tumor oxygenation predicts for the likelihood of distant metastases in human soft tissue sarcoma. Cancer Res (1996) 56:941-3.
The protocol was approved by the Health Research Ethics Board of Alberta-Cancer Committee.

\section{AUTHOR CONTRIBUTIONS}

GS and L-MP contributed to research design. GS and ID conducted experiments; data analysis was carried out by GS, ID, and RL. GS wrote the manuscript; all authors provided feedback and approved the final version.

\section{ACKNOWLEDGMENTS}

Flow cytometry was performed at the University of Alberta, Faculty of Medicine and Dentistry Flow Cytometry Facility, which received financial support from the Faculty of Medicine and Dentistry and the Canadian Foundation for Innovation (CFI) awards to contributing investigators. We thank Nidhi Gupta for assistance in obtaining the breast tumor tissues, and Achim Jungbluth for sharing his protocol for detection of $\gamma \delta \mathrm{Tc}$ by immunohistochemistry prior to its publication.

\section{FUNDING}

This work has been funded by the London Regional Cancer Program, London, ON (Translational Breast Cancer Postdoctoral award to GS), the Cancer Research Society (CRSOG2013 to L-MP and GS), and the Canadian Breast Cancer Foundation (L-MP). Support was also provided by the Sawin-Baldwin Chair in Ovarian Cancer, Dr. Anthony Noujaim Legacy Oncology Chair, and Alberta Innovates Health Solutions Translational Health Chair to LP. ID has been supported by the Queen Elizabeth II Graduate Scholarship, the University of Alberta Doctoral Recruitment Scholarship, and the Alberta Cancer Foundation Antoine Noujaim Scholarship.

\section{SUPPLEMENTARY MATERIAL}

The Supplementary Material for this article can be found online at https://www.frontiersin.org/articles/10.3389/fimmu.2018.01367/ full\#supplementary-material.
6. Brizel DM, Sibley GS, Prosnitz LR, Scher RL, Dewhirst MW. Tumor hypoxia adversely affects the prognosis of carcinoma of the head and neck. Int J Radiat Oncol Biol Phys (1997) 38:285-9. doi:10.1016/S0360-3016(97)00101-6

7. Semenza GL. Defining the role of hypoxia-inducible factor 1 in cancer biology and therapeutics. Oncogene (2010) 29:625-34. doi:10.1038/onc.2009.441

8. Noman MZ, Hasmim M, Messai Y, Terry S, Kieda C, Janji B, et al. Hypoxia: a key player in antitumor immune response. A review in the theme: cellular responses to hypoxia. Am J Physiol Cell Physiol (2015) 309:C569-79. doi:10.1152/ ajpcell.00207.2015

9. Barsoum IB, Hamilton TK, Li X, Cotechini T, Miles EA, Siemens DR, et al. Hypoxia induces escape from innate immunity in cancer cells via increased expression of ADAM10: role of nitric oxide. Cancer Res (2011) 71:7433-41. doi:10.1158/0008-5472.CAN-11-2104

10. Silva-Santos B, Serre K, Norell H. gammadelta T cells in cancer. Nat Rev Immunol (2015) 15:683-91. doi:10.1038/nri3904

11. Wesch D, Peters C, Siegers GM. Human gamma delta T regulatory cells in cancer: fact or fiction? Front Immunol (2014) 5:598. doi:10.3389/fimmu.2014.00598 
12. Lo Presti E, Dieli F, Meraviglia S. Tumor-infiltrating gammadelta T lymphocytes: pathogenic role, clinical significance, and differential programming in the tumor microenvironment. Front Immunol (2014) 5:607. doi:10.3389/fimmu. 2014.00607

13. Chitadze G, Oberg HH, Wesch D, Kabelitz D. The ambiguous role of gammadelta T lymphocytes in antitumor immunity. Trends Immunol (2017) 38:668-78. doi:10.1016/j.it.2017.06.004

14. Guo BL, Liu Z, Aldrich WA, Lopez RD. Innate anti-breast cancer immunity of apoptosis-resistant human gammadelta-T cells. Breast Cancer Res Treat (2005) 93:169-75. doi:10.1007/s10549-005-4792-8

15. Dutta I, Postovit LM, Siegers GM. Apoptosis induced via gamma delta T cell antigen receptor "blocking" antibodies: a cautionary tale. Front Immunol (2017) 8:776. doi:10.3389/fimmu.2017.00776

16. Aggarwal R, Lu J, Kanji S, Das M, Joseph M, Lustberg MB, et al. Human Vgamma2Vdelta2 $\mathrm{T}$ cells limit breast cancer growth by modulating cell survival-, apoptosis-related molecules and microenvironment in tumors. Int J Cancer (2013) 133:2133-44. doi:10.1002/ijc.28217

17. Tafreshi NK, Lloyd MC, Proemsey JB, Bui MM, Kim J, Gillies RJ, et al. Evaluation of CAIX and CAXII expression in breast cancer at varied O2 levels: CAIX is the superior surrogate imaging biomarker of tumor hypoxia. Mol Imaging Biol (2016) 18:219-31. doi:10.1007/s11307-015-0885-x

18. Jin MS, Lee H, Park IA, Chung YR, Im SA, Lee KH, et al. Overexpression of HIFlalpha and CAXI predicts poor outcome in early-stage triple negative breast cancer. Virchows Arch (2016) 469:183-90. doi:10.1007/s00428-0161953-6

19. Tan EY, Yan M, Campo L, Han C, Takano E, Turley H, et al. The key hypoxia regulated gene CAIX is upregulated in basal-like breast tumours and is associated with resistance to chemotherapy. Br J Cancer (2009) 100:405-11. doi:10.1038/ sj.bjc. 6604844

20. Chia SK, Wykoff CC, Watson PH, Han C, Leek RD, Pastorek J, et al. Prognostic significance of a novel hypoxia-regulated marker, carbonic anhydrase IX, in invasive breast carcinoma. J Clin Oncol (2001) 19:3660-8. doi:10.1200/JCO. 2001.19.16.3660

21. Lu J, Aggarwal R, Kanji S, Das M, Joseph M, Pompili V, et al. Human ovarian tumor cells escape gammadelta $\mathrm{T}$ cell recognition partly by down regulating surface expression of MICA and limiting cell cycle related molecules. PLoS One (2011) 6:e23348. doi:10.1371/journal.pone.0023348

22. Groh V, Rhinehart R, Secrist H, Bauer S, Grabstein KH, Spies T. Broad tumor-associated expression and recognition by tumor-derived gamma delta T cells of MICA and MICB. Proc Natl Acad Sci U S A (1999) 96:6879-84. doi:10.1073/pnas.96.12.6879

23. Das H, Groh V, Kuijl C, Sugita M, Morita CT, Spies T, et al. MICA engagement by human Vgamma2Vdelta2 $\mathrm{T}$ cells enhances their antigen-dependent effector function. Immunity (2001) 15:83-93. doi:10.1016/S1074-7613(01)00168-6

24. Bonneville M, O'Brien RL, Born WK. Gammadelta T cell effector functions: a blend of innate programming and acquired plasticity. Nat Rev Immunol (2010) 10:467-78. doi:10.1038/nri2781

25. Bauer S, Groh V, Wu J, Steinle A, Phillips JH, Lanier LL, et al. Activation of NK cells and T cells by NKG2D, a receptor for stress-inducible MICA. Science (1999) 285:727-9. doi:10.1126/science.285.5428.727

26. Siegers GM, Lamb LS Jr. Cytotoxic and regulatory properties of circulating Vdelta1+ gammadelta T cells: a new player on the cell therapy field? Mol Ther (2014) 22:1416-22. doi:10.1038/mt.2014.104

27. Lamb LS Jr, Lopez RD. gammadelta T cells: a new frontier for immunotherapy? Biol Blood Marrow Transplant (2005) 11:161-8. doi:10.1016/j. bbmt.2004.11.015

28. Gomes AQ, Martins DS, Silva-Santos B. Targeting gammadelta T lymphocytes for cancer immunotherapy: from novel mechanistic insight to clinical application. Cancer Res (2010) 70:10024-7. doi:10.1158/0008-5472.CAN$10-3236$

29. Hannani D, Ma Y, Yamazaki T, Déchanet-Merville J, Kroemer G, Zitvogel L. Harnessing gammadelta $\mathrm{T}$ cells in anticancer immunotherapy. Trends Immunol (2012) 33:199-206. doi:10.1016/j.it.2012.01.006

30. Kabelitz D, Wesch D, Pitters E, Zoller M. Potential of human gammadelta T lymphocytes for immunotherapy of cancer. Int J Cancer (2004) 112:727-32. doi:10.1002/ijc. 20445

31. Scheper W, Sebestyen Z, Kuball J. Cancer immunotherapy using gammadeltaT cells: dealing with diversity. Front Immunol (2014) 5:601. doi:10.3389/ fimmu.2014.00601
32. Meraviglia S, Eberl M, Vermijlen D, Todaro M, Buccheri S, Cicero G, et al. In vivo manipulation of Vgamma9Vdelta2 $\mathrm{T}$ cells with zoledronate and low-dose interleukin-2 for immunotherapy of advanced breast cancer patients. Clin Exp Immunol (2010) 161:290-7. doi:10.1111/j.1365-2249.2010. 04167.x

33. Jungbluth AA, Frosina D, Fayad M, Pulitzer MP, Dogan A, Busam KJ, et al. Immunohistochemical detection of $\gamma / \delta \mathrm{T}$ lymphocytes in formalin-fixed paraffin-embedded tissues. Appl Immunohistochem Mol Morphol (2018). doi:10.1097/PAI.0000000000000650

34. Siegers GM, Ribot EJ, Keating A, Foster PJ. Extensive expansion of primary human gamma delta $\mathrm{T}$ cells generates cytotoxic effector memory cells that can be labeled with Feraheme for cellular MRI. Cancer Immunol Immunother (2013) 62:571-83. doi:10.1007/s00262-012-1353-y

35. Holliday DL, Speirs V. Choosing the right cell line for breast cancer research. Breast Cancer Res (2011) 13:215. doi:10.1186/bcr2889

36. Fisher JP, Heuijerjans J, Yan M, Gustafsson K, Anderson J. gammadelta $\mathrm{T}$ cells for cancer immunotherapy: a systematic review of clinical trials. Oncoimmunology (2014) 3:e27572. doi:10.4161/onci.27572

37. Lafont V, Sanchez F, Laprevotte E, Michaud H-A, Gros L, Eliaou J-F, et al. Plasticity of gammadelta $\mathrm{T}$ cells: impact on the anti-tumor response. Front Immunol (2014) 5:622. doi:10.3389/fimmu.2014.00622

38. Carding SR, Egan PJ. Gammadelta T cells: functional plasticity and heterogeneity. Nat Rev Immunol (2002) 2:336-45. doi:10.1038/nri797

39. Vantourout P, Hayday A. Six-of-the-best: unique contributions of gammadelta T cells to immunology. Nat Rev Immunol (2013) 13:88-100. doi:10.1038/ nri3384

40. Trastour C, Benizri E, Ettore F, Ramaioli A, Chamorey E, Pouysségur J, et al. HIF-1alpha and CA IX staining in invasive breast carcinomas: prognosis and treatment outcome. Int J Cancer (2007) 120:1451-8. doi:10.1002/ijc. 22436

41. Ma C, Zhang Q, Ye J, Wang F, Zhang Y, Wevers E, et al. Tumor-infiltrating gammadelta $\mathrm{T}$ lymphocytes predict clinical outcome in human breast cancer. J Immunol (2012) 189(10):5029-36. doi:10.4049/jimmunol.1201892

42. Choi J, Jung WH, Koo JS. Metabolism-related proteins are differentially expressed according to the molecular subtype of invasive breast cancer defined by surrogate immunohistochemistry. Pathobiology (2013) 80:41-52. doi:10.1159/000339513

43. Hidalgo JV, Bronsert P, Orlowska-Volk M, Diaz LB, Stickeler E, Werner M, et al. Histological analysis of gammadelta T lymphocytes infiltrating human triple-negative breast carcinomas. Front Immunol (2014) 5:632. doi:10.3389/ fimmu.2014.00632

44. Dieli F, Poccia F, Lipp M, Sireci G, Caccamo N, Di Sano C, et al. Differentiation of effector/memory Vdelta2 $\mathrm{T}$ cells and migratory routes in lymph nodes or inflammatory sites. J Exp Med (2003) 198:391-7. doi:10.1084/jem.20030235

45. Naldini A, Carraro F, Silvestri S, Bocci V. Hypoxia affects cytokine production and proliferative responses by human peripheral mononuclear cells. J Cell Physiol (1997) 173:335-42. doi:10.1002/(SICI)1097-4652(199712) 173:3<335::AID-JCP5>3.0.CO;2-O

46. Dyugovskaya L, Lavie P, Lavie L. Phenotypic and functional characterization of blood gammadelta T cells in sleep apnea. Am J Respir Crit Care Med (2003) 168:242-9. doi:10.1164/rccm.200210-1226OC

47. Tikhonov I, Deetz CO, Paca R, Berg S, Lukyanenko V, Lim JK, et al. Human Vgamma2Vdelta2 T cells contain cytoplasmic RANTES. Int Immunol (2006) 18:1243-51. doi:10.1093/intimm/dxl055

48. Caux C, Ait-Yahia S, Chemin K, de Bouteiller O, Dieu-Nosjean MC, Homey B, et al. Dendritic cell biology and regulation of dendritic cell trafficking by chemokines. Springer Semin Immunopathol (2000) 22:345-69. doi:10.1007/ s002810000053

49. Sozzani S, Allavena P, Vecchi A, Mantovani A. Chemokines and dendritic cell traffic. J Clin Immunol (2000) 20:151-60. doi:10.1023/A:1006659211340

50. Soria G, Ben-Baruch A. The inflammatory chemokines CCL2 and CCL5 in breast cancer. Cancer Lett (2008) 267:271-85. doi:10.1016/j.canlet.2008. 03.018

51. Hudspeth K, Fogli M, Correia DV, Mikulak J, Roberto A, Della Bella S et al. Engagement of NKp30 on Vdelta1 T cells induces the production of CCL3, CCL4, and CCL5 and suppresses HIV-1 replication. Blood (2012) 119:4013-6. doi:10.1182/blood-2011-11-390153

52. Yamada M, Shiroko T, Kawaguchi Y, Sugiyama Y, Egilmez NK, Chen FA, et al. CD40-CD40 ligand (CD154) engagement is required but not sufficient 
for modulating MHC class I, ICAM-1 and Fas expression and proliferation of human non-small cell lung tumors. Int J Cancer (2001) 92:589-99. doi:10.1002/ijc. 1224

53. Alexandroff AB, Jackson AM, Paterson T, Haley JL, Ross JA, Longo DL, et al. Role for CD40-CD40 ligand interactions in the immune response to solid tumours. Mol Immunol (2000) 37:515-26. doi:10.1016/S0161-5890(00) 00079-1

54. Tong AW, Papayoti MH, Netto G, Armstrong DT, Ordonez G, Lawson JM, et al. Growth-inhibitory effects of CD40 ligand (CD154) and its endogenous expression in human breast cancer. Clin Cancer Res (2001) 7:691-703.

55. Hirano A, Longo DL, Taub DD, Ferris DK, Young LS, Eliopoulos AG, et al. Inhibition of human breast carcinoma growth by a soluble recombinant human CD40 ligand. Blood (1999) 93:2999-3007.

56. Peng G, Wang HY, Peng W, Kiniwa Y, Seo KH, Wang RF. Tumor-infiltrating gammadelta $\mathrm{T}$ cells suppress $\mathrm{T}$ and dendritic cell function via mechanisms controlled by a unique toll-like receptor signaling pathway. Immunity (2007) 27:334-48. doi:10.1016/j.immuni.2007.05.020

57. Barsoum IB, Koti M, Siemens DR, Graham CH. Mechanisms of hypoxiamediated immune escape in cancer. Cancer Res (2014) 74:7185-90. doi:10.1158/ 0008-5472.CAN-14-2598

58. Siemens DR, Hu N, Sheikhi AK, Chung E, Frederiksen LJ, Pross H, et al. Hypoxia increases tumor cell shedding of MHC class I chain-related molecule: role of nitric oxide. Cancer Res (2008) 68:4746-53. doi:10.1158/0008-5472. CAN-08-0054

59. Chitadze G, Lettau M, Luecke S, Wang T, Janssen O, Fürst D, et al. NKG2Dand T-cell receptor-dependent lysis of malignant glioma cell lines by human gammadelta $\mathrm{T}$ cells: modulation by temozolomide and A disintegrin and metalloproteases 10 and 17 inhibitors. Oncoimmunology (2015) 5(4):e1093276. doi:10.1080/2162402X.2015.1093276
60. Deng W, Gowen BG, Zhang L, Wang L, Lau S, Iannello A, et al. Antitumor immunity. A shed NKG2D ligand that promotes natural killer cell activation and tumor rejection. Science (2015) 348:136-9. doi:10.1126/science.1258867

61. Marten A, von Lilienfeld-Toal M, Buchler MW, Schmidt J. Soluble MIC is elevated in the serum of patients with pancreatic carcinoma diminishing gammadelta T cell cytotoxicity. Int J Cancer (2006) 119:2359-65. doi:10.1002/ ijc. 22186

62. Gentles AJ, Newman AM, Liu CL, Bratman SV, Feng W, Kim D, et al. The prognostic landscape of genes and infiltrating immune cells across human cancers. Nat Med (2015) 21:938-45. doi:10.1038/nm.3909

63. Ye J, Ma C, Hsueh EC, Eickhoff CS, Zhang Y, Varvares MA, et al. Tumorderived gammadelta regulatory $\mathrm{T}$ cells suppress innate and adaptive immunity through the induction of immunosenescence. J Immunol (2013) 190:2403-14. doi:10.4049/jimmunol.1202369

64. Ye J, Ma C, Wang F, Hsueh EC, Toth K, Huang Y, et al. Specific recruitment of gammadelta regulatory $\mathrm{T}$ cells in human breast cancer. Cancer Res (2013) 73:6137-48. doi:10.1158/0008-5472.CAN-13-0348

Conflict of Interest Statement: The authors declare that the research was conducted in the absence of any commercial or financial relationships that could be construed as a potential conflict of interest.

Copyright $\odot 2018$ Siegers, Dutta, Lai and Postovit. This is an open-access article distributed under the terms of the Creative Commons Attribution License (CC BY). The use, distribution or reproduction in other forums is permitted, provided the original author(s) and the copyright owner are credited and that the original publication in this journal is cited, in accordance with accepted academic practice. No use, distribution or reproduction is permitted which does not comply with these terms. 